\title{
GAN-Leaks: A Taxonomy of Membership Inference Attacks against Generative Models
}

\author{
Dingfan Chen \\ CISPA Helmholtz Center for Information Security \\ Yang Zhang \\ CISPA Helmholtz Center for Information Security
}

\begin{abstract}
Deep learning has achieved overwhelming success, spanning from discriminative models to generative models. In particular, deep generative models have facilitated a new level of performance in a myriad of areas, ranging from media manipulation to sanitized dataset generation. Despite the great success, the potential risks of privacy breach caused by generative models have not been analyzed systematically. In this paper, we focus on membership inference attack against deep generative models that reveals information about the training data used for victim models. Specifically, we present the first taxonomy of membership inference attacks, encompassing not only existing attacks but also our novel ones. In addition, we propose the first generic attack model that can be instantiated in a large range of settings and is applicable to various kinds of deep generative models. Moreover, we provide a theoretically grounded attack calibration technique, which consistently boosts the attack performance in all cases, across different attack settings, data modalities, and training configurations. We complement the systematic analysis of attack performance by a comprehensive experimental study, that investigates the effectiveness of various attacks w.r.t. model type and training configurations, over three diverse application scenarios (i.e., images, medical data, and location data). ${ }^{1}$
\end{abstract}

\section{CCS CONCEPTS}

- Computing methodologies $\rightarrow$ Machine learning; • Security and privacy;

\section{KEYWORDS}

Membership inference attacks; deep learning; generative models; privacy-preserving machine learning

ACM Reference Format:

Dingfan Chen, Ning Yu, Yang Zhang, and Mario Fritz. 2020. GAN-Leaks: A Taxonomy of Membership Inference Attacks against Generative Models. In

Our code is available at https://github.com/DingfanChen/GAN-Leaks.

Permission to make digital or hard copies of all or part of this work for personal or classroom use is granted without fee provided that copies are not made or distributed for profit or commercial advantage and that copies bear this notice and the full citation on the first page. Copyrights for components of this work owned by others than ACM must be honored. Abstracting with credit is permitted. To copy otherwise, or republish, to post on servers or to redistribute to lists, requires prior specific permission and/or a fee. Request permissions from permissions@acm.org.

CCS '20, November 9-13, 2020, Virtual Event, USA

(C) 2020 Association for Computing Machinery.

ACM ISBN 978-1-4503-7089-9/20/11 ..\$15.00

https://doi.org/10.1145/3372297.3417238

\author{
Ning $\mathrm{Yu}$ \\ University of Maryland, College Park \\ Max Planck Institute for Informatics \\ Mario Fritz \\ CISPA Helmholtz Center for Information Security
}

Proceedings of the 2020 ACM SIGSAC Conference on Computer and Communications Security (CCS '20), November 9-13, 2020, Virtual Event, USA. ACM, New York, NY, USA, 20 pages. https://doi.org/10.1145/3372297.3417238

\section{INTRODUCTION}

Over the last few years, two categories of deep learning techniques have made tremendous progress. The discriminative model has been successfully adopted in various prediction tasks, such as image classification [26, 42, 61, 62] and speech recognition [21, 30]. The generative model, on the other hand, has also gained increasing attention and has delivered appealing applications including photorealistic image synthesis $[20,44,52,70]$, text and sound generation $[4,49,64,65]$, sanitized dataset generation [2, 7, 35, 66, 73], etc. Most of such applications are supported by deep generative models, e.g., the generative adversarial networks (GANs) [3, 10, 20, 23, 36$38,54,59,71]$ and variational autoencoder (VAE) [41, 55, 67].

In line with the growing trend of deep learning in real business, many companies collect and process customer data which is then used to develop deep learning models for commercial use. However, data privacy violations frequently happened due to data misuse with an inappropriate legal basis, e.g., the misuse of $\mathrm{Na}-$ tional Health Service data in the DeepMind project. ${ }^{2}$ Data privacy can also be challenged by malicious users who intend to infer the original training data. The resulting privacy breach would raise serious issues as training data contains sensitive attributes such as diagnosis and income. One such attack is membership inference attack (MIA) [5, 18, 24, 25, 58, 60] which aims to identify if a data record was used to train a machine learning model. Overfitting is the major cause for the feasibility of MIA, as the learned model tends to memorize training inputs and perform better on them.

While numerous literature is dedicated to MIA against discriminative models [33, 45, 48, 50, 58, 60, 68], the attack on generative models has not received equal attention, despite its practical importance. For instance, GANs have been applied to health record data and medical images $[12,19,69]$ whose membership is sensitive as it may reveal a patient's disease history. Moreover, recent works in privacy preserving data sharing $[2,7,11,35,66,73]$ propose to impose (membership) privacy constraints during GANs training for sanitized data generation. Understanding the membership privacy leakage under a practical threat model helps shed light on future research in this area.

Nevertheless, this is a highly challenging task from the adversary side. Unlike discriminative models, the victim generative models

https://news.sky.com/story/google-received-1-6-million-nhs-patients-dataon-an-inappropriate-legal-basis- 10879142 
do not directly provide confidence values about the overfitting of data records, and thus leave little clues for conducting membership inference. In addition, current GAN models inevitably underrepresent certain data samples, i.e., encounter mode dropping and mode collapse, which pose additional difficulty to the attacker.

Unfortunately, none of the existing works [25, 29] provides a generic attack applicable to varying types of generative models. Nor do they report a complete and practical analysis of MIA against deep generative models. For example, Hayes et al. [25] do not consider the realistic situation where the GAN's discriminator is not accessible but only the generator is released. Hilprecht et al. [29] investigate only on small-scale image datasets and do not involve white-box attack against GANs. This motivates our contributions towards a simple and generic approach as well as a more systematic analysis. In general, we make the following contributions in the paper.

Taxonomy of Membership Inference Attacks against Deep Generative Models: We conduct a pioneering study to categorize attack settings against deep generative models. Given the increasing order of the amount of knowledge about a victim model, the settings are benchmarked as (1) full black-box generator, (2) partial black-box generator, (3) white-box generator, and (4) accessible discriminator (full model). In particular, two of the settings, the partial black-box and white-box settings, are of practical value but have not been explored by previous works. We then establish the first taxonomy that comprises the existing and our proposed attacks. See Section 4, Table 1, and Figure 1 for details.

Generic Attack Model and its Novel Instantiated Variants: We propose a simple and generic attack model (Section 5.1) applicable to all the practical settings and various types of deep generative models. More specifically, our generic attack model can be instantiated to a preliminary low-skill attack for the full black-box setting (Section 5.2), a novel black-box optimization-based attack variant in the partial black-box (Section 5.3), as well as a novel quasi-Newton optimization-based variant in the white-box settings (Section 5.4). The consistent effectiveness of our attack model exhibited in all of the aforementioned settings bridges the assumption gap and performance gap between the full black-box attacks and discriminator-accessible attack in previous study [25, 29] through a complete performance spectrum (Section 6.7).

Novel Attack Calibration Technique: To further improve the effectiveness of our attack model, we adjust our approach to each query sample and propose our novel attack calibration technique, which is naturally incorporated in our generic attack framework. Moreover, we prove its near-optimality under a Bayesian perspective. Through extensive experiments, we validate that our attack calibration technique boosts the attack performance noticeably in all cases, across different attack settings, data modalities, and training configurations. See Section 5.6 for detailed explanation and Section 6.6 for experiment results.

Systematic Analysis in Each Setting: We progressively investigate attacks in each setting in the increasing order of amount of knowledge to adversary. See Section 6.3 to Section 6.5 for detailed elaboration. In each setting, our research spans several orthogonal dimensions including three datasets with diverse modalities (Section 6.1), five victim GAN models that were the state-of-the-art at their release time (Section 6.1), two analysis study w.r.t. GAN training configuration (Section 6.2), attack performance gains introduced by attack calibration (Section 5.6 and Section 6.6) and differential private defense (Section 6.8).

\section{RELATED WORK}

Generative Models: Generative models are designed for approximating the probability distribution of the real data. In general, this is done by defining a parametric family of densities and finding the optimal parameters that either maximize the real data likelihood or minimize the divergence between generated and real data distribution. Recent generative models exploit the representation power of deep neural networks for constituting an exceptionally rich parametric family, resulting in tremendous success in modeling high-dimensional data distribution. In this work, we investigate the most widely used deep generative models, namely the generative adversarial networks (GANs) [3, 10, 20, 23, 36-38, 54, 59, 71] and variational autoencoders (VAEs) $[13,14,40]$. Briefly speaking, GANs are trained to minimize the divergence between the generated and real data distribution, while VAEs maximize a lower bound of the real data log-likelihood.

Membership Inference Attacks (MIAs): Shokri et al. [60] specifies the first MIA against discriminative models in the black-box setting, where an attack has access to the victim model's full response (i.e., confidence scores for all classes) for a given input query. They propose to train shadow models that imitate the behavior of the victim model, which generates data to train an attacker model.

Hayes et al. [25] consider MIA against GANs and also propose to retrain a shadow model of the victim model in the black-box case. They then check the discriminator's output scores to query inputs and set a threshold such that all the query inputs with scores larger than the threshold will be classified as in the training set.

Another concurrent study by Hilprecht et al. [29] investigates MIA against both GANs and VAEs. For VAEs, they assume the accessibility of the full model and propose to threshold the $L_{2}$ reconstruction error; For GANs, they only consider the full blackbox setting. Their black-box attack is similar to ours in spirit, as they count the number of generated samples that are inside an $\epsilon$-ball of the query, while we exploit the reconstruction distance instead.

Differential Privacy (DP): Differential privacy [17] is designed to protect the membership privacy of individual samples and is by constructing a defense mechanism against MIA. Recent works propose to train GAN models with differential privacy constraint [2, $7,11,35,66,73]$ and publicize the DP-trained models instead of the raw data, which allows sharing sensitive data while preserving privacy. The differential privacy constraint is fulfilled by replacing the regular stochastic gradient descent with differential private stochastic gradient descent (DP-SGD) [1], which injects calibrated noise in training gradients. As a result, it perturbs data-related objective functions and mitigates inference attacks.

\section{BACKGROUND}

\subsection{Generative Model}

Generative Adversarial Networks (GANs): GANs consist of two neural network modules, a generator $G$ and a discriminator $D$, 
which are trained simultaneously in an adversarial manner. The generator takes random noise $z$ (latent code) as input and generates samples that approximate the training data distribution, while the discriminator receives samples from both the generator and training dataset and is trained to differentiate the two sources. During training, these two modules compete and evolve, such that the generator learns to generate more and more realistic samples aiming at fooling the discriminator, while the discriminator learns to tell the two sources apart more accurately. The training objective can be formulated as

$$
\min _{\theta_{G}} \max _{\theta_{D}} \mathbb{E}_{X \sim P_{\text {data }}}\left[\log \left(D_{\theta_{D}}(x)\right)\right]+\mathbb{E}_{z \sim P_{z}}\left[\log \left(1-D_{\theta_{D}}\left(G_{\theta_{G}}(z)\right)\right)\right]
$$

where $\theta_{G}, \theta_{D}$ denote the parameters of the generator and the discriminator. $P_{\text {data }}$ is the real data distribution, while the $P_{z}$ is the prior distribution of the latent code. The first term in the objective forces the discriminator to output high score given real data sample. The second term makes discriminator output low score on generated samples, while the generator is trained to maximize the discriminator output score. Once the training is done, the discriminator is no longer useful and will normally be discarded. The generator will receive new latent code samples $z$ drawn from the known prior distribution (normally Gaussian) and output the synthetic data samples, which will be collected and used for the downstream task.

Variational Autoencoder (VAE): VAE is another widely used generative framework $[41,55,67]$ consists of an encoder and a decoder, which are cascaded to reconstruct data with pre-defined similarity metrics, e.g. $L_{1} / L_{2}$ loss. The encoder maps data into a latent space, while the decoder maps the encoded latent representation back to the data space. The VAE objective is composed of the reconstruction error and the prior regularization over the latent code distribution. Formally,

$$
\min _{\theta, \phi}-\mathbb{E}_{q_{\phi}(z \mid x)}\left[p_{\theta}(x \mid z)\right]+K L\left(q_{\phi}(z \mid x) \| P_{z}\right)
$$

where $z$ denotes the latent code, $x$ denotes the input data, $q_{\phi}(z \mid x)$ is the probabilistic encoder parameterized by $\phi$ which is introduced to approximate the intractable true posterior, $p_{\theta}(x \mid z)$ represents the probabilistic decoder parameterized by $\theta$, and $K L(\cdot \| \cdot)$ denotes the KL divergence. In practice, $q_{\phi}(z \mid x)$ is always constrained to be uni-modal Gaussian and $z$ is sampled via the reparameterization trick, which results in a closed-form derivation of the second term. Hybrid Model: GANs often suffer from mode collapse and mode dropping issues, i.e., failing to generate appearances relevant to some training samples (low recall), due to the lack of explicit supervison (e.g. data reconstruction) for promoting data mode coverage. VAEs, on the contrary, attain better data coverage but often lack flexible generation capability (low precision). Therefore, a hybrid model, VAEGAN $[8,43]$, is proposed to jointly train a VAE and a GAN, where the VAE decoder and the GAN generator are collapsed into one by sharing trainable parameters. The GAN discriminator is trained to complement the low-level $L_{1}$ or $L_{2}$ reconstruction loss, in order to improve the generation quality of fine-grained details.

\subsection{Membership Inference}

We formulate the membership inference attack as a binary classification task where the attacker aims to classify whether a sample

\begin{tabular}{lccc}
\hline & $\begin{array}{c}\text { Latent } \\
\text { code }\end{array}$ & $\begin{array}{c}\text { Gen- } \\
\text { erator }\end{array}$ & $\begin{array}{c}\text { Dis- }^{-} \\
\text {criminator }\end{array}$ \\
\hline [25] full black-box & $\times$ & $\mathbf{\square}$ & $\times$ \\
[29] full black-box & $\times$ & $\mathbf{a}$ & $\times$ \\
Our full black-box (Section 5.2) & $\times$ & $\mathbf{a}$ & $\times$ \\
Our partial black-box (Section 5.3) & $\checkmark$ & $\mathbf{\square}$ & $\times$ \\
Our white-box (Section 5.4) & $\checkmark$ & $\square$ & $\times$ \\
[25] accessible discriminator (full model) & $\checkmark$ & $\square$ & $\checkmark$ \\
\hline
\end{tabular}

Table 1: Taxonomy of attack settings against GANs over the previous work and ours. $(\times$ : without access; $\checkmark$ : with access; n: black-box; $\square$ : white-box).

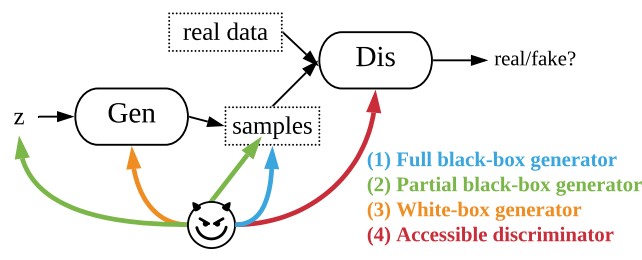

Figure 1: Taxonomy of attack models against GANs. Gen: generator; Dis: discriminator; z: latent code input to Gen.

$x$ has been used to train a victim generative model. Formally, we define

$$
\mathcal{A}:(x, \mathcal{M}(\theta)) \rightarrow\{0,1\}
$$

where the attack model $\mathcal{A}$ output 1 if the attacker infers that the query sample $x$ is included in the training set, and 0 otherwise. $\theta$ denotes the victim model parameters while $\mathcal{M}$ represents the general model publishing mechanism, i.e., type of access available to the attacker. For example, the $\mathcal{M}$ is an identity function for the white-box access case and can be the inference function for the black-box case. For simplicity, we may omit the dependence on $\mathcal{M}$ if the type of access is irrelevant for illustration. With a Bayesian perspective [57], the optimal attacker aims to compute the probability $P\left(x \in D_{\text {train }} \mid x, \theta\right)$ and predict the query sample to be in the training set if the log-likelihood ratio is non-negative, i.e. the query sample is more likely to be contained in the training set than not. Mathematically,

$$
\mathcal{A}(x, \mathcal{M}(\theta))=\mathbb{1}\left[\log \frac{P\left(x \in D_{\text {train }} \mid x, \theta\right)}{P\left(x \notin D_{\text {train }} \mid x, \theta\right)} \geq 0\right]
$$

where $\mathbb{1}(\cdot)$ is the indicator function, and the training set is denoted by $D_{\text {train. }}$. We denote the query sample set as $S=\left\{\left(x_{i}, m_{i}\right)\right\}_{i=1}^{N}$ that contains both training set samples $\left(x_{i} \in D_{\text {train }}, m_{i}=1\right)$ as well as hold-out set samples $\left(x_{i} \notin D_{\text {train }}, m_{i}=0\right)$, where $m$ is the membership indicator variable. The true positive and true negative rate of the attacker can be measure by $\mathbb{E}_{x_{i}}\left[P\left(\mathcal{A}\left(x_{i}, \mathcal{M}(\theta)\right)=1 \mid m_{i}=1\right)\right]$ and $\mathbb{E}_{x_{i}}\left[P\left(\mathcal{A}\left(x_{i}, \mathcal{M}(\theta)\right)=0 \mid m_{i}=0\right)\right]$, respectively.

\section{TAXONOMY}

The attack scenarios can be categorized into either white-box or black-box one. In the white-box setting, the adversary has access to the victim model internals, whereas in the black-box setting, 
the internal workings are unknown to the attackers. For attacks against GANs, we further distinguish the settings based on the accessibility of GANs' components, i.e., the latent code, generator model, and the discriminator model, according to the following criteria: (1) whether the discriminator is accessible, (2) whether the generator is accessible, and (3) whether the latent code is accessible. We elaborate on each category in the following in a decreasing order of the amount of knowledge to attackers. Note that we define the taxonomy in a fully attack-agnostic way, i.e. the attacker can freely decide which part of the available information to use.

\subsection{Accessible Discriminator (Full Model)}

By construction, the discriminator is only used for the adversarial training and normally will be discarded after the training stage is completed. The only scenario in which the discriminator is accessible to the attacker is that the developers publish the whole GAN model along with the source code and allow fine-tuning. In this case, both the discriminator and the generator are accessible to the adversary in a white-box manner. This is the most knowledgeable setting for attackers. And the existing attack methods against discriminative models [60] can be applied to this setting. This setting is also considered in [25], corresponding to the last row in Table 1. In practice, however, the discriminator of a well-trained GAN is discarded without being deployed to APIs, and thus not accessible to attackers. We, therefore, devote less effort to investigating the discriminator and mainly focus on the following practical and generic settings where the attackers only have access to the generator.

\subsection{White-box Generator}

Following the common practice, researchers from the generative modeling community always publish their well-trained generators and code, which allows users to generate new samples and validate the results. This corresponds to the settings that the generator is accessible to the adversary in a white-box manner, i.e. the attackers have access to the internals of the generator. This scenario is also commonly studied in the community of differential privacy [16] and privacy preserving data generation $[2,7,11,35,66,73]$, where people enforce privacy guarantee by training and sharing their generative models instead of sharing the raw private data. Our attack model under this setting can serve as a practical tool for empirically estimating the privacy risk incurred by sharing the differentially private generative models, which offers clear interpretability towards bridging between theory and practice. However, this setting has not been explored by any previous work and is a novel case for constructing a membership inference attack against GANs. It corresponds to the second last row in Table 1 and Section 5.4.

\subsection{Partial Black-box Generator (Known Input-output Pair)}

This is a less knowledgeable setting to attackers where they have no access to the internals of the generator but have access to the latent code of each generated sample. This is a practical setting where the developers retain ownership of their well-trained models while allowing users to control the properties of the generated samples by manipulating the latent code distribution [32], which is a desired feature for application scenarios such as GAN-based

\begin{tabular}{cl}
\hline Notation & Description \\
\hline $\mathcal{A}$ & Attacker \\
$\mathcal{M}$ & model publishing mechanism \\
$D_{\text {train }}$ & Training set of the victim generator \\
$\mathcal{S}$ & Query set \\
$\mathcal{R}$ & Attacker's reconstructor \\
$x$ & Query sample \\
$m$ & Membership indicator variable \\
$z$ & Latent code (input to the generator) \\
$\mathcal{G}_{v}$ & Victim generator \\
$\mathcal{G}_{r}$ & Attacker's reference generator, described in Section 5.6 \\
$\theta_{v}$ & Victim model's parameter \\
$\theta_{r}$ & Attacker's reference model's parameter \\
\hline
\end{tabular}

Table 2: Notations.

image processing [22] and facial attribute editing [27, 37]. This is another novel setting and not considered in previous works [25, 29]. It corresponds to the third last row in Table 1 and Section 5.3.

\subsection{Full Black-box Generator (Known Output Only)}

This is the least knowledgeable setting to attackers where they are passive, i.e., unable to provide input, but are only permitted to access the generated samples set from the well-trained black-box generator. Hayes et al. [25] investigate attacks in this setting by retraining a local copy of the victim model. Hilprecht et al. [29] count the number of generated samples that are inside an $\epsilon$-ball of the query, based on an elaborate design of distance metric. Our idea is similar in spirit to Hilprecht et al. [29] but we score each query by the reconstruction error directly, which does not introduce additional hyperparameter while achieving superior performance. In short, we design a low-skill attack method with a simpler implementation (Section 5.2) that achieves comparable or better performance (Section 6.3). Our attack and theirs correspond to the third, second, and first rows in Table 1, respectively.

\section{ATTACK MODEL}

\subsection{Generic Attack Model}

As mentioned in Section 3.2, the optimal attacker computes the probability $P\left(m_{i}=1 \mid x_{i}, \theta_{v}\right)$. Specifically for the generative model, we make the assumption that this probability should be proportional to the probability that the query sample can be generated by the generator. This assumption holds in general as the generative model is trained to approximate the training data distribution, i.e., $P_{\mathcal{G}_{v}} \approx$ $P_{D_{\text {train }}}$ where $\mathcal{G}_{v}$ denotes the victim generator. And if the probability that the query sample is generated by the victim generator is large, it is more likely that the query sample is used to train the generative model. Formally,

$$
P\left(m_{i}=1 \mid x_{i}, \theta_{v}\right) \propto P_{\mathcal{G}_{v}}\left(x \mid \theta_{v}\right)
$$

However, computing the exact probability is intractable as the distribution of the generated data cannot be represented with an explicit density function. Therefore, we adopt the Parzen window 


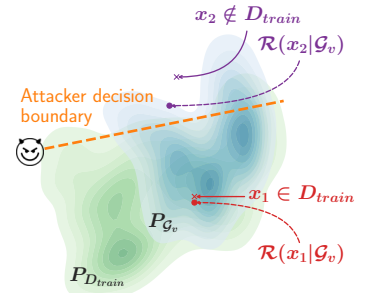

(a) Generic attack model (Section 5.1)

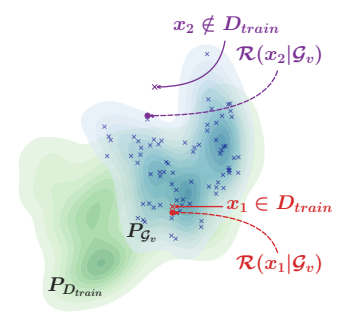

(b) Full black-box attack (Section 5.2)

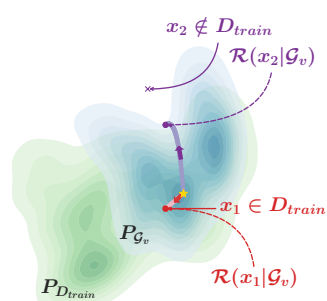

(c) Partial black-box and white-box attack (Section 5.3 and Section 5.4)

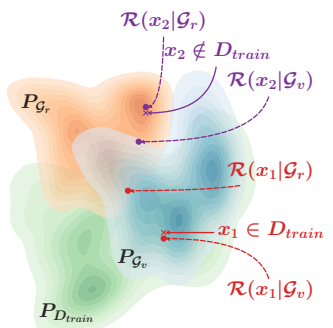

(d) Attack calibration (Section 5.6)

Figure 2: Diagram of our attacks. Mathematical notations refer to Table 2. $P$ represents data distribution. $x_{1}$ belongs to $D_{\text {train }}$ so that it should be better represented by $\mathcal{G}_{v}$ with a smaller distance to its reconstructed copy $\mathcal{R}\left(x_{1} \mid \mathcal{G}_{v}\right)$. $x_{2}$ does not belong to $D_{\text {train }}$ so that it should have a larger distance to its best approximation $\mathcal{R}\left(x_{2} \mid \mathcal{G}_{v}\right)$ in $P_{\mathcal{G}_{v}}$. (a) Our generic attacker set a decision boundary based on the reconstruction distance to infer membership. (b) The best reconstruction is determined over random samples from $P_{\mathcal{G}_{v}}$ while in (c) it is found by optimization on the manifold of $P_{\mathcal{G}_{v}}$. (d) $P_{\mathcal{G}_{r}}$ is a third-party reference GAN distribution where the reconstruction distance is calibrated by the distance between $x$ and $\mathcal{R}\left(x \mid \mathcal{G}_{r}\right)$.

density estimation [15] and approximate the probability as below,

$$
\begin{aligned}
P_{\mathcal{G}_{v}}\left(x \mid \theta_{v}\right) & =\frac{1}{k} \sum_{i=1}^{k} \phi\left(x, \mathcal{G}_{v}\left(z_{i}\right)\right) ; \quad z_{i} \sim P_{z} \\
& \approx \frac{1}{k} \sum_{i=1}^{k} \exp \left(-L\left(x, \mathcal{G}_{v}\left(z_{i}\right)\right)\right) ; \quad z_{i} \sim P_{z}
\end{aligned}
$$

where $\phi(\cdot, \cdot)$ denotes the kernel function, $L(\cdot, \cdot)$ is the general distance metric defined in Section 5.5, and $k$ is the number of samples. Note that this can be further simplified and well approximated using only few samples [9], as all of the terms in the summation of Equation 3, except for a few, will be negligible since $\phi(x, y)$ exponentially decreases with distance between $x, y$.

\subsection{Full Black-box Attack}

We start with the least knowledgeable setting where an attacker only has access to a black-box generator $\mathcal{G}_{v}$. The attacker is allowed no other operation but blindly collecting $k$ samples from $\mathcal{G}_{v}$, denoted as $\left\{\mathcal{G}_{v}(\cdot)_{i}\right\}_{i=1}^{k} \cdot \mathcal{G}_{v}(\cdot)$ indicates that the attacker has neither access nor control over latent code input. We then approximate the probability in Equation 4 using the largest term which is given by the nearest neighbor to $x$ among $\left\{\mathcal{G}_{v}(\cdot)_{i}\right\}_{i=1}^{k}$. Formally,

$$
\mathcal{R}\left(x \mid \mathcal{G}_{v}\right)=\underset{\hat{x} \in\left\{\mathcal{G}_{v}(\cdot)_{i}\right\}_{i=1}^{k}}{\operatorname{argmin}} L(x, \hat{x})
$$

See Figure 2(b) for a diagram. This approximation bound the complete Parzen window from below, but in practice we observe almost no difference when incorporating more terms in the summation for a fixed $k$. However, we find the estimation more sensitive to $k$, and in general a larger $k$ leads to better reconstructions (Figure 10) but at the price of a higher query and computation cost. Throughout the experiments, we consider a practical and limited budget and choose $k$ to be of the same magnitude as the training dataset size.

\subsection{Partial Black-box Attack}

In some practical scenario discussed in Section 4.3, the access to the latent code $z$ is permitted. We then propose to exploit $z$ in order to find a better reconstruction of the query sample and thus improve the $P_{\mathcal{G}_{v}}\left(x \mid \theta_{v}\right)$ estimation. Concretely, the attacker performs an black-box optimization with respect to $z$. Formally,

$$
\mathcal{R}\left(x \mid \mathcal{G}_{v}\right)=\mathcal{G}_{v}\left(z^{*}\right)
$$

where

$$
z^{*}=\underset{z}{\operatorname{argmin}} L\left(x, \mathcal{G}_{v}(z)\right)
$$

Without knowing the internals of $\mathcal{G}_{v}$, the optimization is not differentiable and no gradient information is available. As only the evaluation of function (forward-pass through the generator) is allowed by the access of $\left\{z, \mathcal{G}_{v}(z)\right\}$ pair, we propose to approximate the optimum via the Powell's Conjugate Direction Method [53].

\subsection{White-box Attack}

In the white-box setting, we have the same reconstruction formulation as in Section 5.3. See Figure 2(c) for a diagram. More advantageously to attackers, the reconstruction quality can be further boosted thanks to access to the internals of $\mathcal{G}_{v}$. With access to the gradient information, the optimization problem can be more accurately solved by advanced first-order optimization algorithms [39, 46, 63]. In our experiment, we apply the L-BFGS algorithm for its robustness against suboptimal initialization and its superior convergence rate in comparison to the other methods.

\subsection{Distance Metric}

Our distance metric $L(\cdot, \cdot)$ consists of three terms: the element-wise (pixel-wise) difference term $L_{2}$ targets low-frequency components, the deep image feature term $L_{\text {lpips }}$ (i.e., the Learned Perceptual Image Patch Similarity (LPIPS) metric [72]) targets realism details, and the regularization term penalizes latent code far from the prior 


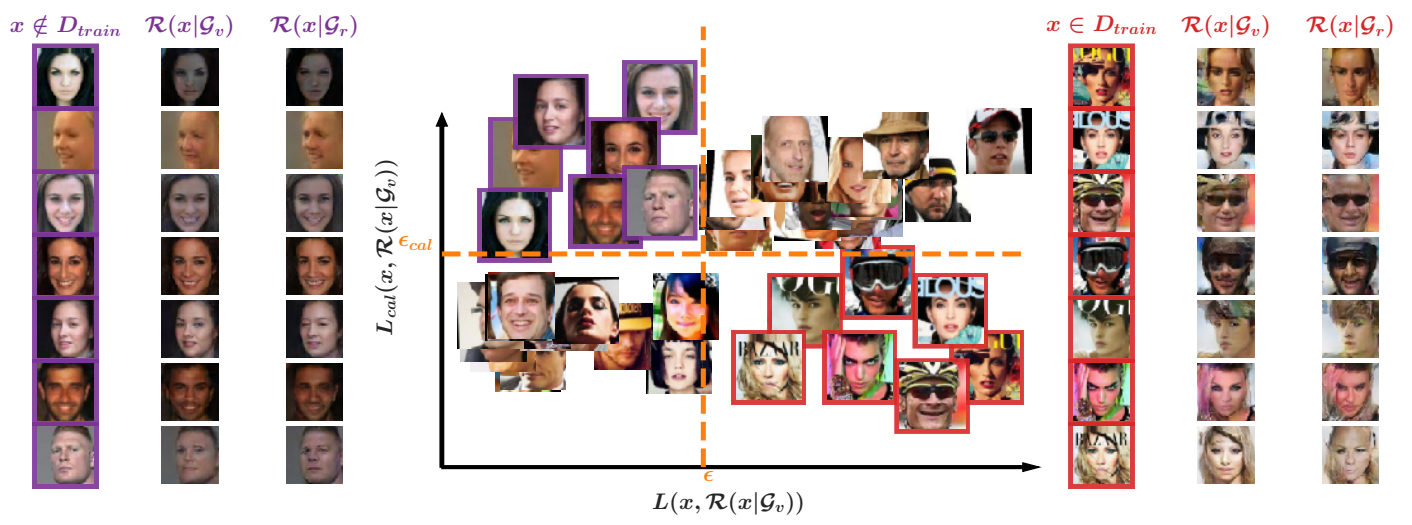

Figure 3: The effectiveness of calibration when attacking PGGAN on CelebA. The $x$ - and $y$-axes respectively represent the distance before $(L)$ and after calibration $\left(L_{\text {cal }}\right)$ between a query sample $x$ and its reconstruction $\mathcal{R}\left(x \mid \mathcal{G}_{v}\right)$. $\epsilon$ and $\epsilon_{\text {cal }}$ are the corresponding thresholds for classification. The false-positive (in purple frame) as well as the false-negative samples (in red frame) before $(L)$ calibration can be corrected by calibration $\left(L_{\text {cal }}\right)$.

distribution. Mathematically,

$$
\begin{aligned}
L\left(x, \mathcal{G}_{v}(z)\right)= & \lambda_{1} L_{2}\left(x, \mathcal{G}_{v}(z)\right)+\lambda_{2} L_{\text {lpips }}\left(x, \mathcal{G}_{v}(z)\right) \\
& +\lambda_{3} L_{\mathrm{reg}}(z)
\end{aligned}
$$

where

$$
\begin{gathered}
L_{2}\left(x, \mathcal{G}_{v}(z)\right)=\left\|x-\mathcal{G}_{v}(z)\right\|_{2}^{2} \\
L_{\mathrm{reg}}(z)=\left(\|z\|_{2}^{2}-\operatorname{dim}(z)\right)^{2}
\end{gathered}
$$

$\lambda_{1}, \lambda_{2}$ and $\lambda_{3}$ are used to enable/disable and balance the order of magnitude of each loss term. For non-image data, $\lambda_{2}=0$ because LPIPS is no longer applicable. For full black-box attack, $\lambda_{3}=0$ as the constraint $z \sim P_{z}$ is satisfied by the sampling process.

\subsection{Attack Calibration}

We noticed that the reconstruction error is query-dependent, i.e., some query samples are more (less) difficult to reconstruct due to their intrinsically more (less) complicated representations, regardless of which generator is used. In this case, the reconstruction error is dominated by the representations rather than by the membership clues. We, therefore, propose to mitigate the query dependency by first independently training a reference GAN $\mathcal{G}_{r}$ with a relevant but disjoint dataset, and then calibrating our base reconstruction error according to the reference reconstruction error. Formally,

$$
L_{\text {cal }}\left(x, \mathcal{R}\left(x \mid \mathcal{G}_{v}\right)\right)=L\left(x, \mathcal{R}\left(x \mid \mathcal{G}_{v}\right)\right)-L\left(x, \mathcal{R}\left(x \mid \mathcal{G}_{r}\right)\right)
$$

with $\mathcal{R}$ the reconstruction. As demonstrated in Figure 3, we show in the up-left quadrant the query samples in purple frame that are classified as in $D_{\text {train }}$ by $L$ and as not in $D_{\text {train }}$ by $L_{\text {cal }}$. They are false-positive to $L$ but are corrected to true-negative by $L_{\text {cal }}$. On the other hand, we show in the bottom-right quadrant the query samples in red frame that are classified as not in $D_{\text {train }}$ by $L$ and as in $D_{\text {train }}$ by $L_{\text {cal }}$. They are false-negative to $L$ but are corrected to true-positive by $L_{\text {cal }}$. We compare all these samples, their reconstructions from the victim generator $\mathcal{G}_{v}$, and their reconstructions from the reference generator $\mathcal{G}_{r}$ on the two sides of the plot. The false-positive samples by $L$ on the left-hand side are those with less complicated appearances such that their reconstruction errors are not high given arbitrary generators. In contrast, the false-negative samples by $L$ on the right-hand side are those with more complicated appearances such that their reconstruction errors are high given arbitrary generators. Our calibration can effectively mitigate these two types of misclassification that depend on sample representations.

As discussed in Section 3.2, the optimal attacker aims to compute the membership probability

$$
P\left(m_{i}=1 \mid \theta_{v}, x_{i}\right)=\mathbb{E}_{S}\left[P\left(m_{i}=1 \mid \theta_{v}, x_{i}, S\right)\right]
$$

Specifically, inferring the membership of the query sample $x_{i}$ amounts to approximating the value of $P\left(m_{i}=1 \mid \theta_{v}, x_{i}, S\right)$ [57]. We show that our calibrated loss well approximate this probability by the following theorem, whose proof is provided in Appendix.

TheOREM 5.1. Given the victim model with parameter $\theta_{v}$, a query dataset $S$, the membership probability of a query sample $x_{i}$ is well approximated by the sigmoid of minus calibrated reconstruction error.

$$
P\left(m_{i}=1 \mid \theta_{v}, x_{i}, S\right) \approx \sigma\left(-L_{c a l}\left(x_{i}, \mathcal{R}\left(x_{i} \mid \mathcal{G}_{v}\right)\right)\right.
$$

And the optimal attack is equivalent to

$$
\mathcal{A}\left(x_{i}, \mathcal{M}\left(\theta_{v}\right)\right)=\mathbb{1}\left[L_{c a l}\left(x_{i}, \mathcal{R}\left(x_{i} \mid \mathcal{G}_{v}\right)\right)<\epsilon\right]
$$

i.e., the attacker checks whether the calibrated reconstruction error of the query sample $x_{i}$ is smaller than a threshold $\epsilon$.

In the white-box case, the reference model has the same architecture as the victim model as this information is accessible to the attacker. In the full black-box and partial black-box settings, $\mathcal{G}_{r}$ has irrelevant network architectures to $\mathcal{G}_{v}$, which is fixed across attack scenarios. The optimization on the well-trained $\mathcal{G}_{r}$ is the same as on the white-box $\mathcal{G}_{v}$. See Figure 2(d) for a diagram, and Section 6.6 for implementation details.

\section{EXPERIMENTS}

Based on the proposed taxonomy, we present the most comprehensive evaluation to date on the membership inference attacks 


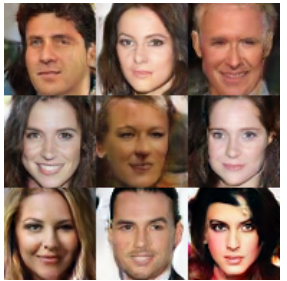

(a) PGGAN

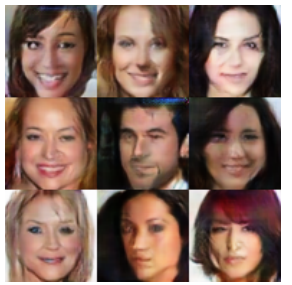

(b) WGANGP

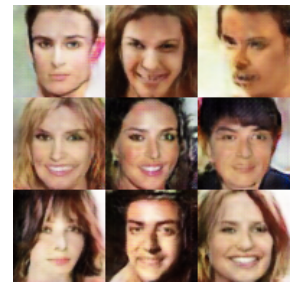

(c) DCGAN

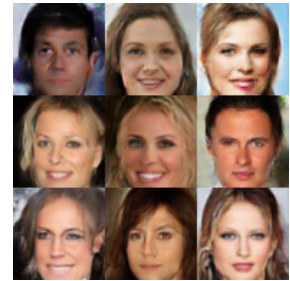

(d) VAEGAN

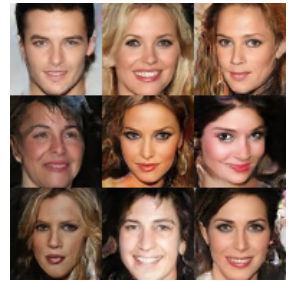

(e) PGGAN w/ DP

Figure 4: Generated images from different victim GAN models trained on CelebA.

against deep generative models. While prior studies have singled out few data sets from constraint domains on selected models, our evaluation includes three diverse datasets, five different generative models, and systematic analysis of attack vectors - including more viable threat models. Via this approach, we present key discoveries, that connect for the first time the effectiveness of the attacks to the model types, data sets, and training configuration.

\subsection{Setup}

Datasets: We conduct experiments on three diverse modalities of datasets covering images, medical records, and location check-ins, which are considered with a high risk of privacy breach.

CelebA [47] is a large-scale face attributes dataset with 200k RGB images. Images are aligned to each other based on facial landmarks, which benefits GAN performance. We select at most 20k images, center-crop them, and resize them to $64 \times 64$ before GAN training.

MIMIC-III [34] is a public Electronic Health Records (EHR) database containing medical records of 46, 520 intensive care unit (ICU) patients. We follow the same procedure as in [12] to pre-process the data, where each patient is represented by a 1071-dimensional binary feature vector. We filter out patients with repeated vector presentations and yield 41,307 unique samples.

Instagram New-York [6] contains Instagram users' check-ins at various locations in New York at different time stamps from 2013 to 2017 . We filter out users with less than 100 check-ins and yield 34, 336 remaining samples. For sample representation, we first select 2, 024 evenly-distributed time stamps. We then concatenate the longitude and latitude values of the check-in location at each time stamp, and yield a 4048-dimensional vector for each sample. The longitude and latitude values are either retrieved from the dataset or linearly interpolated from the available neighboring time stamps. We then perform zero-mean normalization before GAN training.

Victim GAN Models: We select PGGAN [36], WGANGP [23], DCGAN [54], MEDGAN [12], and VAEGAN [8] into the victim model set, considering their pleasing performance on generating images and/or other data representations.

It is important to guarantee the high quality of well-trained GANs because attackers are more likely to target high-quality GANs with practical effectiveness. We noticed previous works [25, 29] only show qualitative results of their victim GANs. In particular, Hayes et al. [25] did not show visually pleasing generated results on the Labeled Faces in the Wild (LFW) dataset [31]. Rather, we present better qualitative results of different GANs on CelebA (Figure 4),

\begin{tabular}{cccccc|c}
\hline $\begin{array}{c}\text { PG- } \\
\text { GAN }\end{array}$ & $\begin{array}{c}\text { WGAN- } \\
\text { GP }\end{array}$ & $\begin{array}{c}\text { DC- } \\
\text { GAN }\end{array}$ & $\begin{array}{c}\text { VAE- } \\
\text { GAN }\end{array}$ & $\begin{array}{c}\text { SOTA } \\
\text { ref }\end{array}$ & $\begin{array}{c}\text { PGGAN } \\
\text { w/ DP }\end{array}$ \\
\hline FID & 14.86 & 24.26 & 35.40 & 53.08 & 7.40 & 15.63 \\
\hline
\end{tabular}

Table 3: FID for different GAN models trained on CelebA. "SOTA ref" represents the state-of-the-art result reported in [10] over $128 \times 128$ ImageNet ILSVRC 2012 dataset [56]. “w/ DP” represents the GAN model with DP privacy protection [1] (see Section 6.8).

and further present the corresponding quantitative evaluation in terms of Fréchet Inception Distance (FID) metric [28] (Table 3). A smaller FID indicates the generated image set is more realistic and closer to real-world data distribution. We show that our GAN models are in a reasonable range to the state of the art.

Attack Evaluation: The proposed membership inference attack is formulated as a binary classification given a threshold $\epsilon$ in Equation 14 . Through varying $\epsilon$, we measure the area under the receiver operating characteristic curve (AUCROC) to evaluate the attack performance.

\subsection{Analysis Study}

We first list two dimensions of analysis study across attack settings. There are also some other dimensions specifically for the white-box attack, which are elaborated in Section 6.5.

6.2.1 GAN Training Set Size. Training set size is highly related to the degree of overfitting of GAN training. A GAN model trained with a smaller size tends to more easily memorize individual training images and is thus more vulnerable to membership inference attack. Moreover, training set size is the main factor that affects the privacy cost computation for differential privacy. Therefore, we evaluate the attack performance w.r.t. training set size. We exclude DCGAN and VAEGAN from evaluation since they yield unstable training for small training sets.

6.2.2 Random v.s. Identity-based Selection for GAN Training Set. There are different levels of difficulty for membership inference attack. For example, CelebA contains person identity information and we can design attack difficulty by composing GAN training set based on identity or not. In one case, we include all images of the selected individuals for training(identity). In the other case, we ignore identity information and randomly select images for 


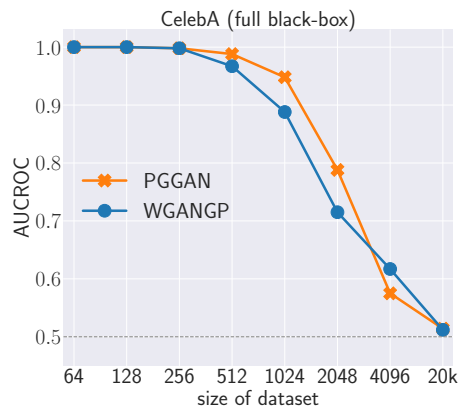

(a)

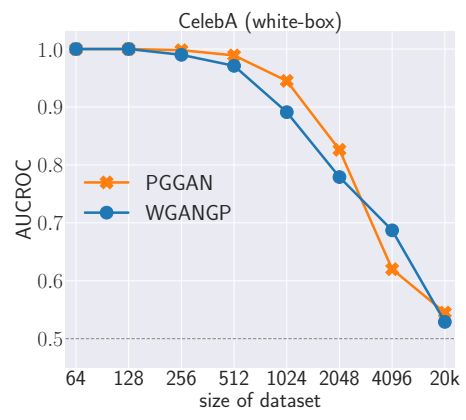

(d)

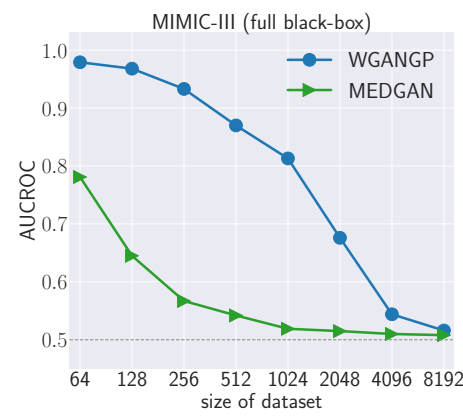

(b)

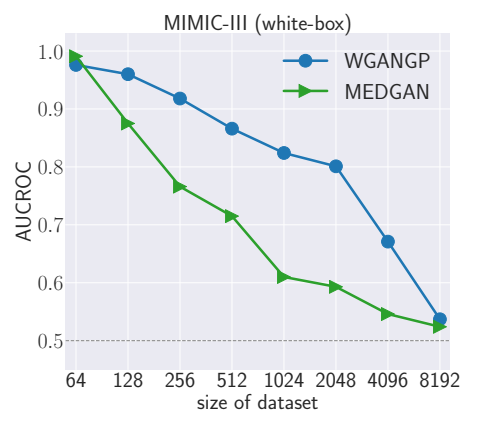

(e)

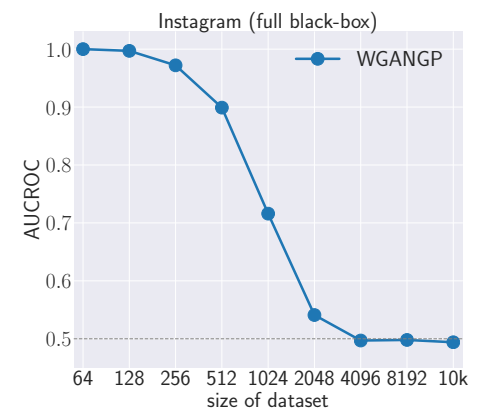

(c)

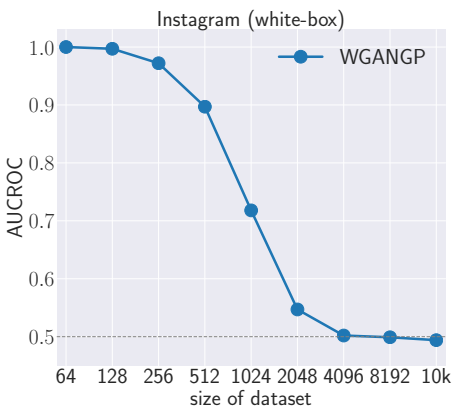

(f)

Figure 5: Full black-box attack (the first row) and white-box attack (the second row) performance w.r.t. GAN training set size.

training(random), i.e., it is possible that some images for an individual are in the training dataset while some are not. The former case is relatively easier to attackers with a larger margin between membership image set and non-membership image set. In line with previous work [25], we evaluate these two kinds of training set selection schemes on CelebA for a complete and fair comparison.

\subsection{Evaluation on Full Black-box Attack}

We start with evaluating our preliminary low-skill black-box attack model in order to gain a sense of the difficulty of the whole problem.

6.3.1 Performance w.r.t. GAN Training Set Size. Figure 5(a) to Figure 5(c) plot the attack performance against different GAN models on the three datasets. As shown in the plots, the attack performs sufficiently well when the training set is small for all three datasets. For instance, on CelebA, when the training set contains up to 512 images, attacker's AUCROC on both PGGAN and WGANGP are above 0.95 . This indicates an almost perfect attack and a serious privacy breach. For larger training sets, however, the attacks become less effective as the degree of overfitting decreases and GAN's capability shifts from memorization to generalization. It is also consistent with the objective of GAN, i.e., to model the underlying distribution of the whole population instead of fitting a particular data sample. Hence, the collection of more data for GAN training can reduce privacy breach of individual samples. Moreover, PGGAN becomes more vulnerable than WGANGP on CelebA when the training size becomes larger. WGANGP is consistently more vulnerable than MEDGAN on MIMIC-III regardless of training size.
6.3.2 Performance w.r.t. GAN Training Set Selection. Figure 6(a) shows the attack performance w.r.t. training set selection schemes on four victim GAN models when fixing the training set size. We observe that, consistently, all the GAN models are more vulnerable when the training set is selected based on identity. Hence, more attention needs to be paid to an identity-based privacy breach, which is more likely to happen than an instance-based privacy breach. Moreover, when compared among different victim GAN models, DCGAN and VAEGAN are more resistant against the full black-box attack with AUCROC only marginally above 0.5 (random guess baseline). This may be attributed to the poor generation quality of DCGAN and VAEGAN (Table 3), as it indicates that a certain amount of data samples can not be well represented by the victim model and thus the reconstruction error will be a less accurate approximation of the true membership probability in Equation 2.

\subsection{Evaluation on Partial Black-box Attack}

6.4.1 Performance w.r.t. GAN Training Set Selection. Figure 6(b) shows the comparison on four victim GAN models. Similar to the case of the full black-box attack (Section 6.3), we find that all models become more vulnerable to identity-based selection. Still, DCGAN is the most resistant victim against membership inference in both training set selection schemes, probably due to its inferior generation quality.

6.4.2 Comparison to Full Black-box Attack. Comparing between Figure 6(a) and Figure 6(b), the attack performance against each GAN model consistently and significantly improves from black-box 


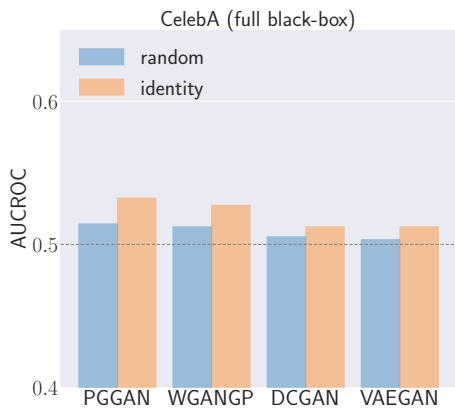

(a)

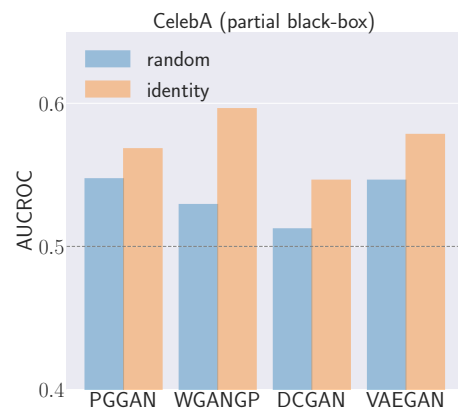

(b)

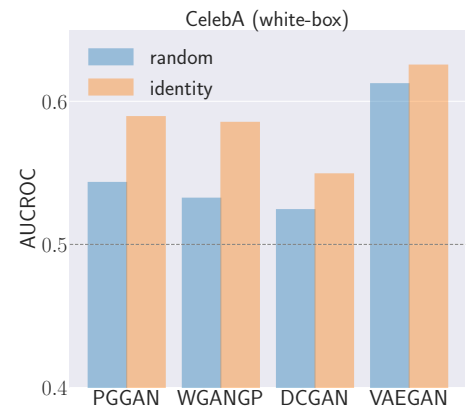

(c)

Figure 6: Attack performance on the random v.s. identity-based training set selection (CelebA with size=20k).

setting to partial black-box setting. We attribute this improvement to a better reconstruction of query samples found by the attacker via optimization. Hence, we conclude that providing the input interface to a generator suffers from an increased privacy risk.

\subsection{Evaluation on White-box Attack}

We further investigate the case where the victim generator is published in a white-box manner. This scenario is commonly studied in the field of privacy preserving data generation $[2,7,11,35,66,73]$, where our approach can serve as a simple and interpretable framework for empirically quantifying the privacy leakage. As the optimization in the white-box attack involves more technical details, we conduct additional analysis study and sanity check in this setting. See Appendix C. 1 for more details.

6.5.1 Performance w.r.t. GAN Training Set Size. Figure 5(d) to Figure 5(f) plot the attack performance against different GAN models on the three datasets when varying training set size. We find that the attack becomes less effective as the training set becomes larger, similar to that in the black-box setting. For CelebA, the attack remains effective for 20k training samples, while for MIMIC-III and Instagram, this number decreases to 8192 and 2048, respectively. The strong similarity between the member and non-member in these two non-image datasets increases the difficulty of attack, which explains the deteriorated effectiveness of the attack model.

6.5.2 Performance w.r.t. GAN Training Set Selection. Figure 6(c) shows the comparisons against four victim GAN models. Our attack is much more effective when composing GAN training set according to identity, which is similar to those in the full and partial black-box settings.

6.5.3 Comparison to Full and Partial Black-box Attacks. For membership inference attack, it is an important question whether or to what extent the white-box attack is more effective than the black-box ones. For discriminative (classification) models, recent literature reports that the state-of-the-art black-box attack performs almost as well as the white-box attack [51, 57]. In contrast, we find that against generative models the white-box attack is much more effective. Comparisons across subfigures in Figure 6 show that the AUCROC values increase by at least 0.03 when changing from full black-box to white-box setting. Compared to the partial black-box attack, the white-box attack achieves noticeably better performance against PGGAN and VAEGAN. Moreover, conducting the white-box attack requires much less computation cost than conducting the partial black-box attack. Therefore, we conclude that publicizing model parameters (white-box setting) does incur high privacy breach risk.

\subsection{Performance Gain from Attack Calibration}

We perform calibration on all the settings. Note that for full and partial black-box settings, attackers do not have prior knowledge of victim model architectures. We thus train a PGGAN on LFW face dataset [31] and use it as the generic reference model for calibrating all victim models trained on CelebA in the black-box settings. Similarly, for MIMIC-III, we use WGANGP as the reference model for MedGAN and vice versa. In other words, we have to guarantee that our calibrated attacks strictly follow the black-box assumption.

Figure 7 compares attack performance on CelebA before and after applying calibration. The AUCROC values are improved consistently across all the GAN architectures in all the settings. In general, the white-box attack calibration yields the greatest performance gain. Moreover, the improvement is especially significant when attacking against VAEGAN, as the AUCROC value increases by 0.2 after applying calibration.

Figure 8 compares attack performance on the other two nonimage datasets. The performance is also consistently boosted for all training set sizes after calibration.

\subsection{Comparison to Baseline Attacks}

We compare our calibrated attack to two recent membership inference attack baselines: Hayes et al. [25] (denoted as LOGAN) and Hilprecht et al. [29] (denoted as MC, standing for their proposed Monte Carlo sampling method). As described in our taxonomy (Section 4), LOGAN includes a full black-box attack model and a discriminator-accessible attack model against GANs. The latter is regarded as the most knowledgeable but unrealistic setting because the discriminator in GAN is usually not accessible in practice. But we still compare to both settings for the completeness of our taxonomy and experiments. MC includes a full black-box attack against GANs and a full-model-accessible attack against VAEs. We evaluate 


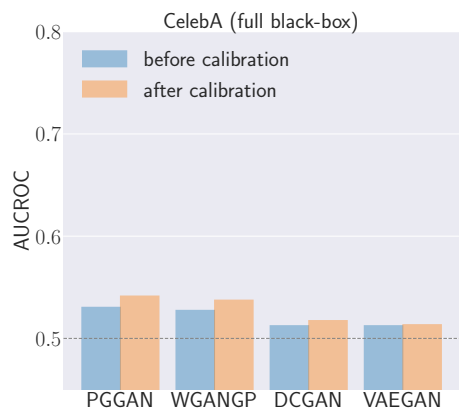

(a)

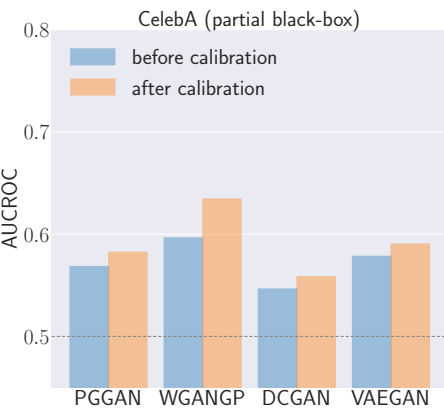

(b)

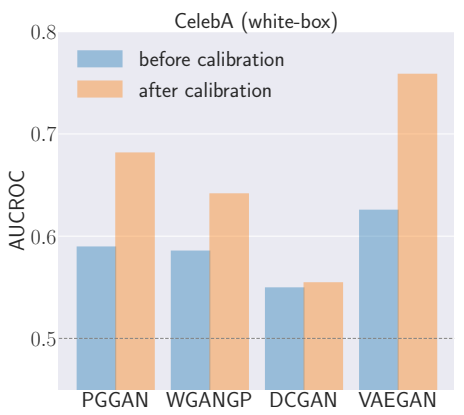

(c)

Figure 7: Attack performance before and after calibration on CelebA (size=20k).

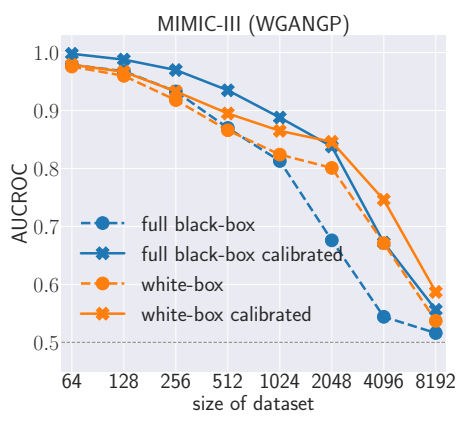

(a)

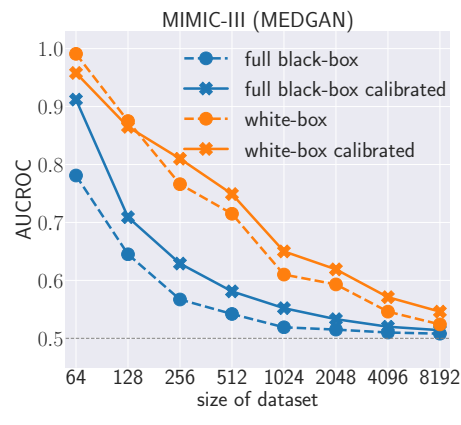

(b)

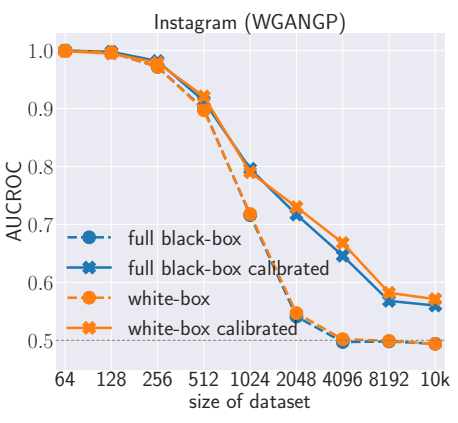

(c)

Figure 8: Attack performance before and after calibration for non-image datasets w.r.t. GAN training set sizes.

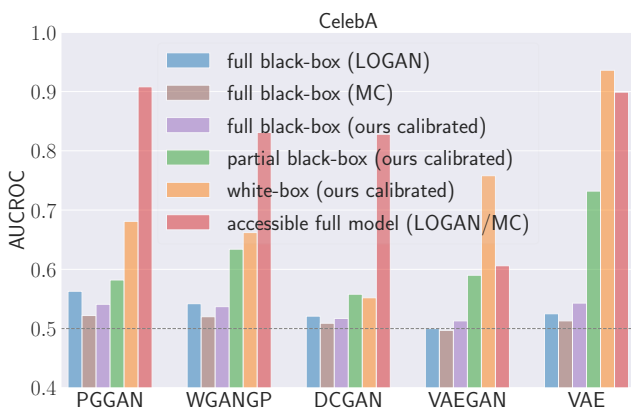

Figure 9: Comparison of different attacks on CelebA. See Table 12 in Appendix for quantitative results.

our generic attack model on both GANs and VAEs for a complete comparison, though we mainly focus on GANs in this work. Note that, to the best of our knowledge, there does not exist any attack against GANs in the partial black-box or white-box settings.

Figure 9, Figure 10, and Figure 11 show the comparisons, considering several datasets, victim models, training set sizes, numbers of query images (full black-box), and different attack settings. We skip MC on the non-image datasets as it is not directly applicable in terms of their distance calculation. Our findings are as follows.

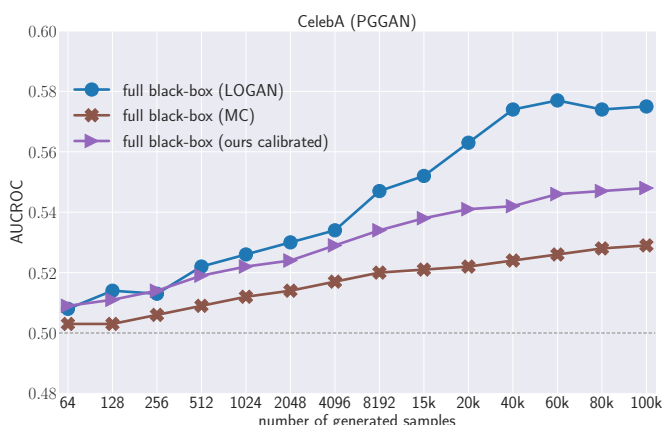

Figure 10: Full black-box attack performance against PGGAN on CelebA w.r.t. $k$ in Equation 5, the number of generated samples. See Table 14 in Appendix for quantitative results.

In the black-box setting, our low-skill attack consistently outperforms MC and outperforms LOGAN on the non-image datasets. It also achieves comparable performance to LOGAN on CelebA but with a much simpler and learning-free implementation.

Our white-box and even partial black-box attacks consistently outperform the other full black-box attacks. Hence, publicizing the 


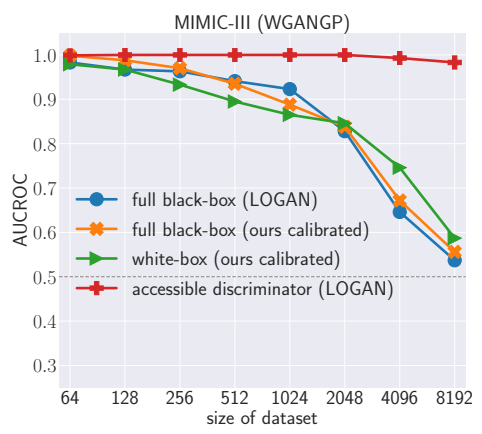

(a)

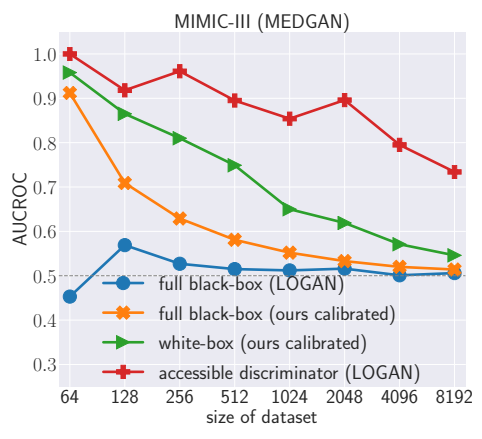

(b)

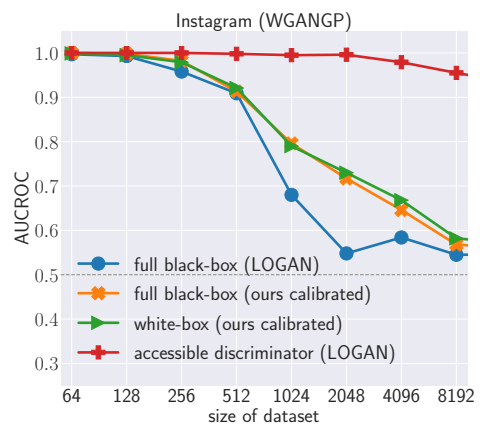

(c)

Figure 11: Comparison of different attacks on the other two non-image datasets w.r.t. GAN training set size. See Table 13 in Appendix for quantitative results.

generator or even just the input to the generator can lead to a considerably higher risk of privacy breach. With a complete spectrum of performance across settings, they bridge the performance gap between the highly constrained full black-box attack and the unrealistic discriminator-accessible attack. Moreover, our proposed white-box attack model is of practical value for the differential privacy community.

Assuming the accessibility of discriminator (full model) normally results in the most effective attack. This can be explained by the fact that the discriminator is explicitly trained to maximize the margin between training set (membership samples) and generated set (a subset of non-membership samples), which eventually yields very accurate confidence scores for membership inference. Surprisingly, our calibrated white-box attack even outperforms baseline methods in more knowledgeable settings, i.e., LOGAN (accessible discriminator) for VAEGAN and MC (accessible full model) for VAE. This shows that when data coverage is explicitly enforced, which probably leads to overfitting and data memorization if not properly regularized, our attack models are highly effectively and achieve superior performance with a more realistic assumption.

\subsection{Defense}

We investigate the most effective defense mechanism against MIA to date that is applicable to GANs [7, 25, 66, 73], i.e., the differential private (DP) stochastic gradient descent [1]. The algorithm can be summarized into two steps. First, the per-sample gradient computed at each training iteration is clipped by its $L_{2}$ norm with a pre-defined threshold. Subsequently, calibrated random noise is added to the gradient in order to inject stochasticity for protecting privacy. In this scheme, however, privacy protection is at the cost of computational complexity and utility deterioration, i.e., slower training and lower generation quality.

We conduct attacks against PGGAN on CelebA, which has been defended by DP. We skip the other cases because DP always deteriorates generation quality to an unacceptable level. The hyperparameters are selected through the grid search. We fix the norm threshold to 1.0 (average gradient norm magnitude during pretraining) and the noise scale to $10^{-4}$ (the largest value with which we obtain samples of good visual quality). However, this results in

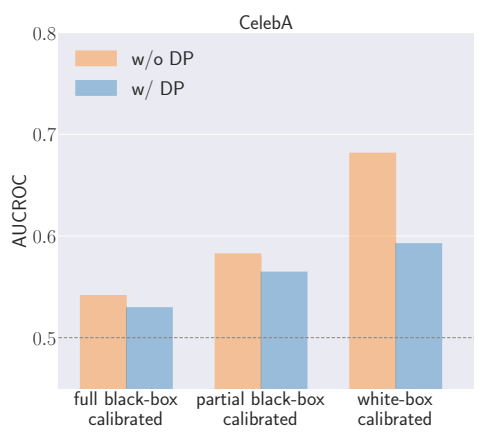

(a)

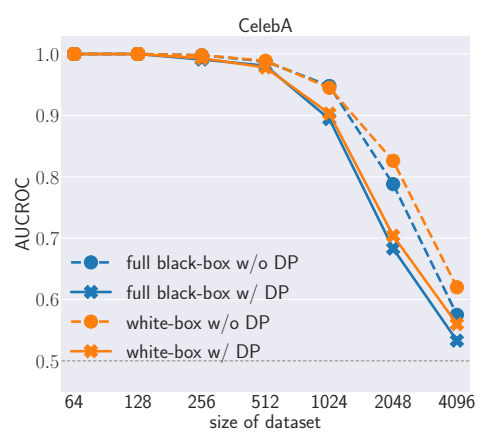

(b)

Figure 12: (a) Attack performance against PGGAN on CelebA with or without DP defense. (b)Attack performance against PGGAN on CelebA with or without DP defense, w.r.t. GAN training set size. We fix all the other control factors (training iterations, batch size, noise scale, norm threshold) apart from training set size, which results in less privacy guarantee for a smaller dataset.

high $\epsilon$ values ( $>10^{10}$ for a default value of $\delta=10^{-5}$ ), while it still reduces the effectiveness of the membership inference attack. 
Figure 12(a) and Figure 12(b) depict the attack performance in different settings. We observe a consistent decrease in AUCROC in all the settings. Therefore, DP is effective in general against our attack. However, applying DP into training leads to a much higher computation cost (10× slower) in practice due to the per-sample gradient modification. Moreover, DP results in a deterioration of GAN utility, which is witnessed by an increasing FID (comparing the last and second columns in Table 3). Moreover, for obtaining a pleasing level of utility, the noise scale has to be limited to a small value, which, in turn, cannot defend the membership inference attack completely. For example, for all the settings, our attack still achieves better performance than the random guess baseline $($ AUCROC $=0.5)$.

\subsection{Summary}

Before ending this section, we show a few insights over the experiment results and list practical considerations relevant to the deployment of GANs and potential privacy breaches.

- The vulnerability of models under MIA heavily relies on the attackers' knowledge about victim models. Releasing the discriminator (full model) results in an exceptionally high risk of privacy breach, which can be explained by the fact that the discriminator had full access to the training data and thus easily memorizes the private information about the training data. Similarly, the release of the generator and/or the control over the input noise $z$ also incurs a relatively high privacy risk.

- The vulnerability of different generative models under MIA varies. Although the effectiveness of MIA mainly depends on the generation quality of victim models, the objective function and training paradigm also play important roles. Specifically, when data reconstruction is explicitly formulated in the training objective to improve data mode coverage, e.g. in VAEGAN and VAE, the resulting models become highly vulnerable to MIA.

- A smaller training dataset leads to a higher risk of revealing information of individual samples. In particular, if the magnitude of training set size is less than $10 k$ where most existing GAN models have sufficient modeling capacity for overfitting to individual sample, the membership privacy is highly likely to be compromised once the GAN model and/or its generated sample set is released. This causes special concern when dealing with real-world privacy sensitive datasets (e.g. medical records), which typically contain very limited data samples.

- Differential private defense on GAN training is effective against practical MIA, but at the cost of high computation burden and deteriorated generation quality.

\section{CONCLUSION}

We have established the first taxonomy of membership inference attacks against GANs, with which we hope to benchmark research in this direction in the future. We have also proposed the first generic attack model based on reconstruction, which is applicable to all the settings according to the amount of the attacker's knowledge about the victim model. In particular, the instantiated attack variants in the partial black-box and white-box settings are another novelty that bridges the assumption gap and performance gap in the previous work [25, 29]. In addition, we proposed a novel theoretically grounded attack calibration technique, which consistently improve the attack performance in all cases. Comprehensive experiments show consistent effectiveness and a broad spectrum of performance in a variety of setups spanning diverse dataset modalities, various victim models, two directions of analysis study, attack calibration, as well as differential privacy defense, which conclusively provide a better understanding of privacy risks associated with deep generative models.

\section{ACKNOWLEDGMENTS}

This work is partially funded by the Helmholtz Association within the projects "Trustworthy Federated Data Analytics" (TFDA) (funding number ZT-I-OO1 4) and "Protecting Genetic Data with Synthetic Cohorts from Deep Generative Models" (PRO-GENE-GEN) (funding number ZT-I-PF-5-23).

\section{REFERENCES}

[1] Martin Abadi, Andy Chu, Ian Goodfellow, Brendan McMahan, Ilya Mironov, Kunal Talwar, and Li Zhang. 2016. Deep Learning with Differential Privacy. In ACM SIGSAC Conference on Computer and Communications Security (CCS). ACM, 308-318.

[2] Gergely Acs, Luca Melis, Claude Castelluccia, and Emiliano De Cristofaro. 2017. Differentially Private Mixture of Generative Neural Networks. In International Conference on Data Mining (ICDM). IEEE, 715-720.

[3] Martin Arjovsky, Soumith Chintala, and Léon Bottou. 2017. Wasserstein Generative Adversarial Networks. In International Conference on Machine Learning (ICML). JMLR, 214-223.

[4] Sanjeev Arora, Rong Ge, Yingyu Liang, Tengyu Ma, and Yi Zhang. 2017. Generalization and Equilibrium in Generative Adversarial Nets (GANs). In International Conference on Machine Learning (ICML). JMLR, 224-232.

[5] Michael Backes, Pascal Berrang, Mathias Humbert, and Praveen Manoharan. 2016. Membership Privacy in MicroRNA-based Studies. In ACM SIGSAC Conference on Computer and Communications Security (CCS). ACM, 319-330.

[6] Michael Backes, Mathias Humbert, Jun Pang, and Yang Zhang. 2017. walk2friends: Inferring Social Links from Mobility Profiles. In ACM SIGSAC Conference on Computer and Communications Security (CCS). ACM, 1943-1957.

[7] Brett K Beaulieu-Jones, Zhiwei Steven Wu, Chris Williams, Ran Lee, Sanjeev P Bhavnani, James Brian Byrd, and Casey S Greene. 2019. Privacy-Preserving Generative Deep Neural Networks Support Clinical Data Sharing. Circulation: Cardiovascular Quality and Outcomes 12, 7 (2019), e005122.

[8] Apratim Bhattacharyya, Mario Fritz, and Bernt Schiele. 2019. "Best-of-ManySamples" Distribution Matching. CoRR abs/1909.12598 (2019).

[9] Oren Boiman, Eli Shechtman, and Michal Irani. 2008. In Defense of NearestNeighbor based Image Classification. In IEEE Conference on Computer Vision and Pattern Recognition (CVPR). IEEE.

[10] Andrew Brock, Jeff Donahue, and Karen Simonyan. 2019. Large Scale GAN Training for High Fidelity Natural Image Synthesis. In International Conference on Learning Representations (ICLR)

[11] Dingfan Chen, Tribhuvanesh Orekondy, and Mario Fritz. 2020. GS-WGAN: A Gradient-Sanitized Approach for Learning Differentially Private Generators. CoRR abs/2006.08265 (2020).

[12] Edward Choi, Siddharth Biswal, Bradley Malin, Jon Duke, Walter F. Stewart, and Jimeng Sun. 2018. Generating Multi-label Discrete Patient Records using Generative Adversarial Networks. CoRR abs/1703.06490 (2018).

[13] Laurent Dinh, David Krueger, and Yoshua Bengio. 2015. NICE: Non-linear Independent Components Estimation. CoRR abs/1410.8516 (2015).

[14] Laurent Dinh, Jascha Sohl-Dickstein, and Samy Bengio. 2017. Density Estimation using Real NVP. In International Conference on Learning Representations (ICLR).

[15] Richard O Duda, Peter E Hart, and David G Stork. 2012. Pattern Classification. John Wiley \& Sons.

[16] Cynthia Dwork, Frank McSherry, Kobbi Nissim, and Adam Smith. 2006. Calibrating Noise to Sensitivity in Private Data Analysis. In Theory of Cryptography Conference (TCC). Springer, 265-284.

[17] Cynthia Dwork and Aaron Roth. 2014. The Algorithmic Foundations of Differential Privacy. Now Publishers Inc.

[18] Cynthia Dwork, Adam D. Smith, Thomas Steinke, Jonathan Ullman, and Salil P. Vadhan. 2015. Robust Traceability from Trace Amounts. In Annual Symposium 
on Foundations of Computer Science (FOCS). IEEE, 650-669.

[19] Maayan Frid-Adar, Eyal Klang, Michal Amitai, Jacob Goldberger, and Hayit Greenspan. 2018. Synthetic Data Augmentation using GAN for Improved Liver Lesion Classification. In IEEE International Symposium on Biomedical Imaging (ISBI). IEEE, 289-293.

[20] Ian Goodfellow, Jean Pouget-Abadie, Mehdi Mirza, Bing Xu, David Warde-Farley, Sherjil Ozair, Aaron Courville, and Yoshua Bengio. 2014. Generative Adversarial Nets. In Annual Conference on Neural Information Processing Systems (NIPS). NIPS, $2672-2680$.

[21] Alex Graves, Abdel rahman Mohamed, and Geoffrey E. Hinton. 2013. Speech Recognition with Deep Recurrent Neural Networks. In IEEE International Conference on Acoustics, Speech and Signal Processing (ICASSP). IEEE, 6645-6649.

[22] Jinjin Gu, Yujun Shen, and Bolei Zhou. 2019. Image Processing Using Multi-Code GAN Prior. CoRR abs/1912.07116 (2019)

[23] Ishaan Gulrajani, Faruk Ahmed, Martin Arjovsky, Vincent Dumoulin, and Aaron C. Courville. 2017. Improved Training of Wasserstein GANs. In Annual Conference on Neural Information Processing Systems (NIPS). NIPS, 5767-5777.

[24] Inken Hagestedt, Yang Zhang, Mathias Humbert, Pascal Berrang, Haixu Tang, XiaoFeng Wang, and Michael Backes. 2019. MBeacon: Privacy-Preserving Beacons for DNA Methylation Data. In Network and Distributed System Security Symposium (NDSS). Internet Society.

[25] Jamie Hayes, Luca Melis, George Danezis, and Emiliano De Cristofaro. 2019. LOGAN: Evaluating Privacy Leakage of Generative Models Using Generative Adversarial Networks. Symposium on Privacy Enhancing Technologies Symposium (2019).

[26] Kaiming He, Xiangyu Zhang, Shaoqing Ren, and Jian Sun. 2016. Deep Residual Learning for Image Recognition. In IEEE Conference on Computer Vision and Pattern Recognition (CVPR). IEEE, 770-778.

[27] Zhenliang He, Wangmeng Zuo, Meina Kan, Shiguang Shan, and Xilin Chen. 2018. AttGAN: Facial Attribute Editing by Only Changing What You Want. CoRR $a b s / 1711.10678$ (2018)

[28] Martin Heusel, Hubert Ramsauer, Thomas Unterthiner, Bernhard Nessler, and Sepp Hochreiter. 2017. GANs Trained by a Two Time-Scale Update Rule Converge to a Local Nash Equilibrium. In Annual Conference on Neural Information Processing Systems (NIPS). NIPS, 6626-6637.

[29] Benjamin Hilprecht, Martin Härterich, and Daniel Bernau. 2019. Monte Carlo and Reconstruction Membership Inference Attacks against Generative Models. Symposium on Privacy Enhancing Technologies Symposium (2019).

[30] Geoffrey Hinton, Li Deng, Dong Yu, George Dahl, Abdel rahman Mohamed, Navdeep Jaitly, Andrew Senior, Vincent Vanhoucke, Patrick Nguyen, Brian Kingsbury, et al. 2012. Deep Neural Networks for Acoustic Modeling in Speech Recognition. IEEE Signal Processing Magazine 29 (2012).

[31] Gary B Huang, Marwan Mattar, Tamara Berg, and Eric Learned-Miller. 2008 Labeled Faces in the Wild: A Database for Studying Face Recognition in Unconstrained Environments.

[32] Ali Jahanian, Lucy Chai, and Phillip Isola. 2019. On the "Steerability" of Generative Adversarial Networks. CoRR abs/1907.07171 (2019).

[33] Jinyuan Jia, Ahmed Salem, Michael Backes, Yang Zhang, and Neil Zhenqiang Gong. 2019. MemGuard: Defending against Black-Box Membership Inference Attacks via Adversarial Examples. In ACM SIGSAC Conference on Computer and Communications Security (CCS). ACM, 259-274.

[34] Alistair EW Johnson, Tom J Pollard, Lu Shen, H Lehman Li-wei, Mengling Feng Mohammad Ghassemi, Benjamin Moody, Peter Szolovits, Leo Anthony Celi, and Roger G Mark. 2016. MIMIC-III, A Freely Accessible Critical Care Database. Scientific Data 3 (2016), 160035

[35] James Jordon, Jinsung Yoon, and Mihaela van der Schaar. 2019. PATE-GAN Generating Synthetic Data with Differential Privacy Guarantees. OpenReview (2019)

[36] Tero Karras, Timo Aila, Samuli Laine, and Jaakko Lehtinen. 2018. Progressive Growing of GANs for Improved Quality, Stability, and Variation. In International Conference on Learning Representations (ICLR).

[37] Tero Karras, Samuli Laine, and Timo Aila. 2019. A Style-Based Generator Architecture for Generative Adversarial Networks. In IEEE Conference on Computer Vision and Pattern Recognition (CVPR). IEEE, 4401-4410.

[38] Tero Karras, Samuli Laine, Miika Aittala, Janne Hellsten, Jaakko Lehtinen, and Timo Aila. 2020. Analyzing and Improving the Image Quality of StyleGAN In IEEE Conference on Computer Vision and Pattern Recognition (CVPR). IEEE, 8107-8116.

[39] Diederik P. Kingma and Jimmy Ba. 2015. Adam: A Method for Stochastic Optimization. In International Conference on Learning Representations (ICLR)

[40] Diederik P. Kingma and Prafulla Dhariwal. 2018. Glow: Generative Flow with Invertible 1x1 Convolutions. In Annual Conference on Neural Information Processing Systems (NeurIPS). NeurIPS, 10236-10245.

[41] Diederik P. Kingma and Max Welling. 2014. Auto-Encoding Variational Bayes. In International Conference on Learning Representations (ICLR)

[42] Alex Krizhevsky, Ilya Sutskever, and Geoffrey E. Hinton. 2012. ImageNet Classification with Deep Convolutional Neural Networks. In Annual Conference on Neural Information Processing Systems (NIPS). NIPS, 1106-1114.
[43] Anders Boesen Lindbo Larsen, Søren Kaae Sønderby, Hugo Larochelle, and Ole Winther. 2016. Autoencoding beyond Pixels Using a Learned Similarity Metric. In International Conference on Machine Learning (ICML). JMLR, 1558-1566.

[44] Chuan Li and Michael Wand. 2016. Precomputed Real-Time Texture Synthesis with Markovian Generative Adversarial Networks. In European Conference on Computer Vision (ECCV). Springer, 702-716.

[45] Zheng Li and Yang Zhang. 2020. Label-Leaks: Membership Inference Attack with Label. CoRR abs/2007.15528 (2020).

[46] Dong C Liu and Jorge Nocedal. 1989. On the Limited Memory BFGS Method for Large Scale Optimization. Mathematical Programming 45, 1-3 (1989), 503-528.

[47] Ziwei Liu, Ping Luo, Xiaogang Wang, and Xiaoou Tang. 2015. Deep Learning Face Attributes in the Wild. In IEEE International Conference on Computer Vision (ICCV). IEEE, 3730-3738.

[48] Yunhui Long, Vincent Bindschaedler, Lei Wang, Diyue Bu, Xiaofeng Wang, Haixu Tang, Carl A. Gunter, and Kai Chen. 2018. Understanding Membership Inferences on Well-Generalized Learning Models. CoRR abs/1802.04889 (2018).

[49] Soroush Mehri, Kundan Kumar, Ishaan Gulrajani, Rithesh Kumar, Shubham Jain, Jose Sotelo, Aaron C. Courville, and Yoshua Bengio. 2017. SampleRNN: An Unconditional End-to-End Neural Audio Generation Model. In International Conference on Learning Representations (ICLR).

[50] Luca Melis, Congzheng Song, Emiliano De Cristofaro, and Vitaly Shmatikov. 2019. Exploiting Unintended Feature Leakage in Collaborative Learning. In IEEE Symposium on Security and Privacy (S\&P). IEEE, 497-512.

[51] Milad Nasr, Reza Shokri, and Amir Houmansadr. 2019. Comprehensive Privacy Analysis of Deep Learning: Passive and Active White-box Inference Attacks against Centralized and Federated Learning. In IEEE Symposium on Security and Privacy (S\&P). IEEE, 1021-1035.

[52] Deepak Pathak, Philipp Krähenbühl, Jeff Donahue, Trevor Darrell, and Alexei A. Efros. 2016. Context Encoders: Feature Learning by Inpainting. In IEEE Conference on Computer Vision and Pattern Recognition (CVPR). IEEE, 2536-2544.

[53] Michael JD Powell. 1964. An Efficient Method for Finding the Minimum of a Function of Several Variables without Calculating Derivatives. Comput. 7. 7, 2 (1964), 155-162.

[54] Alec Radford, Luke Metz, and Soumith Chintala. 2015. Unsupervised Representation Learning with Deep Convolutional Generative Adversarial Networks. CoRR $a b s / 1511.06434$ (2015).

[55] Danilo Jimenez Rezende, Shakir Mohamed, and Daan Wierstra. 2014. Stochastic Backpropagation and Approximate Inference in Deep Generative Models. CoRR abs/1401.4082 (2014).

[56] Olga Russakovsky, Jia Deng, Hao Su, Jonathan Krause, Sanjeev Satheesh, Sean Ma, Zhiheng Huang, Andrej Karpathy, Aditya Khosla, Michael Bernstein, Alexander C. Berg, and Li Fei-Fei. 2015. ImageNet Large Scale Visual Recognition Challenge. CoRR abs/1409.0575 (2015)

[57] Alexandre Sablayrolles, Matthijs Douze, Cordelia Schmid, Yann Ollivier, and Hervé Jégou. 2019. White-box vs Black-box: Bayes Optimal Strategies for Membership Inference. In International Conference on Machine Learning (ICML). JMLR, 5558-5567.

[58] Ahmed Salem, Yang Zhang, Mathias Humbert, Pascal Berrang, Mario Fritz, and Michael Backes. 2019. ML-Leaks: Model and Data Independent Membership Inference Attacks and Defenses on Machine Learning Models. In Network and Distributed System Security Symposium (NDSS). Internet Society.

[59] Tim Salimans, Ian J. Goodfellow, Wojciech Zaremba, Vicki Cheung, Alec Radford, and Xi Chen. 2016. Improved Techniques for Training GANs. In Annual Conference on Neural Information Processing Systems (NIPS). NIPS, 2226-2234.

[60] Reza Shokri, Marco Stronati, Congzheng Song, and Vitaly Shmatikov. 2017. Membership Inference Attacks Against Machine Learning Models. In IEEE Symposium on Security and Privacy (S\&P). IEEE, 3-18.

[61] Karen Simonyan and Andrew Zisserman. 2015. Very Deep Convolutional Networks for Large-Scale Image Recognition. In International Conference on Learning Representations (ICLR).

[62] Christian Szegedy, Wei Liu, Yangqing Jia, Pierre Sermanet, Scott E. Reed, Dragomir Anguelov, Dumitru Erhan, Vincent Vanhoucke, and Andrew Rabinovich. 2015. Going Deeper with Convolutions. In IEEE Conference on Computer Vision and Pattern Recognition (CVPR). IEEE, 1-9.

[63] Tijmen Tieleman and Geoffrey Hinton. 2012. Lecture 6.5-rmsprop: Divide the Gradient by a Running average of its Recent Magnitude. COURSERA: Neural Networks for Machine Learning 4, 2 (2012), 26-31.

[64] Aaron van den Oord, Sander Dieleman, Heiga Zen, Karen Simonyan, Oriol Vinyals, Alex Graves, Nal Kalchbrenner, Andrew Senior, and Koray Kavukcuoglu. 2016. WaveNet: A Generative Model for Raw Audio. CoRR abs/1609.03499 (2016).

[65] Oriol Vinyals, Alexander Toshev, Samy Bengio, and Dumitru Erhan. 2015. Show and Tell: A Neural Image Caption Generator. In IEEE Conference on Computer Vision and Pattern Recognition (CVPR). IEEE, 3156-3164.

[66] Liyang Xie, Kaixiang Lin, Shu Wang, Fei Wang, and Jiayu Zhou. 2018. Differentially Private Generative Adversarial Network. CoRR abs/1802.06739 (2018).

[67] Xinchen Yan, Jimei Yang, Kihyuk Sohn, and Honglak Lee. 2016. Attribute2Image: Conditional Image Generation from Visual Attributes. In European Conference on Computer Vision (ECCV). Springer, 776-791. 
[68] Samuel Yeom, Irene Giacomelli, Matt Fredrikson, and Somesh Jha. 2018. Privacy Risk in Machine Learning: Analyzing the Connection to Overfitting. In IEEE Computer Security Foundations Symposium (CSF). IEEE, 268-282.

[69] Xin Yi, Ekta Walia, and Paul Babyn. 2019. Generative Adversarial Network in Medical Imaging: A Review. Medical Image Analysis (2019), 101552.

[70] Ning Yu, Connelly Barnes, Eli Shechtman, Sohrab Amirghodsi, and Michal Lukác 2019. Texture Mixer: A Network for Controllable Synthesis and Interpolation of Texture. In IEEE Conference on Computer Vision and Pattern Recognition (CVPR). IEEE, 12164-12173.

[71] Ning Yu, Ke Li, Peng Zhou, Jitendra Malik, Larry Davis, and Mario Fritz. 2020 Inclusive GAN: Improving Data and Minority Coverage in Generative Models. In European Conference on Computer Vision (ECCV). Springer.

[72] Richard Zhang, Phillip Isola, Alexei A. Efros, Eli Shechtman, and Oliver Wang 2018. The Unreasonable Effectiveness of Deep Features as a Perceptual Metric In IEEE Conference on Computer Vision and Pattern Recognition (CVPR). IEEE, $586-595$.

[73] Xinyang Zhang, Shouling Ji, and Ting Wang. 2018. Differentially Private Releasing via Deep Generative Model (Technical Report). CoRR abs/1801.01594 (2018)

\section{A PROOF}

Theorem 5.1. Given the victim model with parameter $\theta_{v}$, a query dataset $S$, the membership probability of a query sample $x_{i}$ is well approximated by the sigmoid of minus calibrated reconstruction error.

$$
P\left(m_{i}=1 \mid \theta_{v}, x_{i}, S\right) \approx \sigma\left(-L_{c a l}\left(x_{i}, \mathcal{R}\left(x_{i} \mid \mathcal{G}_{v}\right)\right)\right.
$$

And the optimal attack is equivalent to

$$
\mathcal{A}\left(x_{i}, \mathcal{M}\left(\theta_{v}\right)\right)=\mathbb{1}\left[L_{c a l}\left(x_{i}, \mathcal{R}\left(x_{i} \mid \mathcal{G}_{v}\right)\right)<\epsilon\right]
$$

i.e., the attacker checks whether the calibrated reconstruction error of the query sample $x_{i}$ is smaller than a threshold $\epsilon$.

Proof. By applying the Bayes rule and the property of sigmoid function $\sigma$, the membership probability can be rewritten as follows [57]

$$
P\left(m_{i}=1 \mid \theta_{v}, x_{i}, S\right)=\sigma\left(\log \left(\frac{P\left(\theta_{v} \mid m_{i}=1, x_{i}, S_{-i}\right) P\left(m_{i}=1\right)}{P\left(\theta_{v} \mid m_{i}=0, x_{i}, S_{-i}\right) P\left(m_{i}=0\right)}\right)\right)
$$

where $S_{-i}=S \backslash\left(x_{i}, m_{i}\right)$, i.e., the whole query set except the query sample $x_{i}$.

Assuming independence of samples in $\mathcal{S}$ while applying Bayes rule and Product rule, we obtain the following posterior approximation

$$
\begin{aligned}
P\left(\theta_{v} \mid S\right) & \propto \prod_{\left\{j \mid m_{j}=1\right\}} P\left(x_{j} \mid \theta_{v}\right) P\left(\theta_{v}\right) \\
& \propto \exp \left(-\sum_{j} m_{j} \cdot l\left(x_{j}, \theta_{v}\right)\right)
\end{aligned}
$$

with $l\left(x_{j}, \theta_{v}\right)=L\left(x_{j}, \mathcal{R}\left(x \mid \mathcal{G}_{v}\right)\right)$ for brevity. The Equation 18 means that the probability of a certain model parameter is determined by its i.i.d. training set samples. Subsequently, by assuming a uniform prior of the model parameter over the whole parameter space and plug in the results from Equation 4 we obtain Equation 19. By normalizing the posterior in Equation 19, we obtain

$$
\begin{aligned}
& P\left(\theta_{v} \mid m_{i}=1, x_{i}, S_{-i}\right)=\frac{\exp \left(-\sum_{j} m_{j} \cdot l\left(x_{j}, \theta_{v}\right)\right)}{\int_{\theta^{\prime}} \exp \left(-\sum_{j} m_{j} \cdot l\left(x_{j}, \theta^{\prime}\right)\right) d_{\theta^{\prime}}} \\
& P\left(\theta_{v} \mid m_{i}=0, x_{i}, S_{-i}\right)=\frac{\exp \left(-\sum_{j \neq i} m_{j} \cdot l\left(x_{j}, \theta_{v}\right)\right)}{\int_{\theta^{\prime}} \exp \left(-\sum_{j \neq i} m_{j} \cdot l\left(x_{j}, \theta^{\prime}\right)\right) d_{\theta^{\prime}}}
\end{aligned}
$$

with the following ratio:

$$
\frac{P\left(\theta_{v} \mid m_{i}=1, x_{i}, S\right)}{P\left(\theta_{v} \mid m_{i}=0, x_{i}, S\right)}=\frac{\exp \left(-l\left(x_{i}, \theta_{v}\right)\right)}{\int_{\theta^{\prime}} \exp \left(-l\left(x_{i}, \theta^{\prime}\right)\right) P\left(\theta^{\prime} \mid S_{-i}\right) d_{\theta^{\prime}}}
$$

where

$$
P\left(\theta \mid S_{-i}\right)=\frac{\exp \left(-\sum_{j \neq i} m_{j} \cdot l\left(x_{j}, \theta\right)\right)}{\int_{\theta^{\prime}} \exp \left(-\sum_{j \neq i} m_{j} \cdot l\left(x_{j}, \theta^{\prime}\right)\right) d_{\theta^{\prime}}}
$$

Putting things together, we have

$$
\begin{aligned}
P\left(m_{i}=1 \mid \theta_{v}, x_{i}, S\right)=\sigma[ & \log \left(\frac{P\left(m_{i}=1\right)}{P\left(m_{i}=0\right)}\right)-l\left(x_{i}, \theta_{v}\right) \\
& \left.-\log \left(\int_{\theta^{\prime}} \exp \left(-l\left(x_{i}, \theta^{\prime}\right)\right) P\left(\theta^{\prime} \mid S_{-i}\right) d_{\theta^{\prime}}\right)\right]
\end{aligned}
$$

The first term is equivalent to the log ratio of the prior probability, i.e., the fraction of training data in the query set. In most of our experiments, we use a balanced split which makes this term vanish. Thus, only the second and last term will affect the attacker prediction. Next, we investigate the last term. By applying Jensen's inequality, we can bound the last term from above.

$$
\begin{aligned}
-\log \left(\int_{\theta^{\prime}} \exp \left(-l\left(x_{j}, \theta^{\prime}\right)\right) P\left(\theta^{\prime} \mid S_{-i}\right) d_{\theta^{\prime}}\right) & =-\log \mathbb{E}_{\theta^{\prime}} \exp \left(-l\left(x_{i}, \theta^{\prime}\right)\right) \\
& \leq-\mathbb{E}_{\theta^{\prime}} \log \exp \left(-l\left(x_{i}, \theta^{\prime}\right)\right) \\
& =\mathbb{E}_{\theta^{\prime}} l\left(x_{i}, \theta^{\prime}\right)
\end{aligned}
$$

Additionally, we can obtain the lower bound by taking the optimimum over the full parameter space, i.e.

$$
\begin{aligned}
-\log \left(\int_{\theta^{\prime}} \exp \left(-l\left(x_{j}, \theta^{\prime}\right)\right) P\left(\theta^{\prime} \mid S_{-i}\right) d_{\theta^{\prime}}\right) & \geq-\log \max _{\theta^{\prime}} \exp \left(-l\left(x_{i}, \theta^{\prime}\right)\right) \\
& =\min _{\theta^{\prime}} l\left(x_{i}, \theta^{\prime}\right)
\end{aligned}
$$

Under the assumption of a highly peaked posterior, e.g. uni-modal Gaussian [57], we can well approximate this quantity by using one sample, i.e. using one reference model that is not trained on the query sample. Formally,

$$
\begin{aligned}
P\left(m_{i}=1 \mid \theta_{v}, x_{i}, S\right) & \approx \sigma\left[-l\left(x_{i}, \theta_{v}\right)+l\left(x_{i}, \theta_{r}\right)\right] \\
& =\sigma\left[-L\left(x, \mathcal{R}\left(x \mid \mathcal{G}_{v}\right)\right)+L\left(x, \mathcal{R}\left(x \mid \mathcal{G}_{r}\right)\right]\right. \\
& =\sigma\left[-L_{\text {cal }}\left(x, \mathcal{R}\left(x \mid \mathcal{G}_{v}\right)\right]\right.
\end{aligned}
$$

where the dependence on $S, \theta_{v}$ is absorbed in the calibrated distance $L_{\text {cal }}\left(x, \mathcal{R}\left(x \mid \mathcal{G}_{v}\right)\right)$.

Hence, the optimal attacker classifies $x_{i}$ as in the training set if the membership probability is sufficiently large, i.e., $L_{\text {cal }}\left(x, \mathcal{R}\left(x \mid \mathcal{G}_{v}\right)\right)$ is sufficiently small (than a threshold), following from the nondecreasing property of $\sigma$.

\section{B EXPERIMENT SETUP}

\section{B.1 Hyper-parameter Setting}

We fix $k$ to be $20 \mathrm{k}$ for evaluating the full black-box attacks. We set $\lambda_{1}=1.0, \lambda_{2}=0.2, \lambda_{3}=0.001$ for our partial black-box and whitebox attack on CelebA, and set $\lambda_{1}=1.0, \lambda_{2}=0.0, \lambda_{3}=0.0$ for the other cases. The maximum number of iterations for optimization are set to be 1000 for our white-box attack and 10 for our partial black-box attack. 


\section{B.2 Model Architectures}

We use the official implementations of the victim GAN models. ${ }^{3} \mathrm{We}$ re-implement WGANGP model with a fully-connected structure for non-image datasets. The network architecture is summarized in Table 4 . The depth of both the generator and discriminator is set to 5. The dimension of the hidden layer is fix to be 512 . We use ReLU as the activation function for the generator and Leaky ReLU with $\alpha=0.2$ for the discriminator, except for the output layer where either the sigmoid or identity function is used.

\begin{tabular}{l|l|l}
\hline $\begin{array}{l}\text { Generator } \\
\text { (MIMIC-III) }\end{array}$ & $\begin{array}{l}\text { Generator } \\
\text { (Instagram) }\end{array}$ & $\begin{array}{l}\text { Discriminator } \\
\text { (MIMIC-III and Instagram) }\end{array}$ \\
\hline FC (512) & FC (512) & FC (512) \\
ReLU & ReLU & LeakyReLU (0.2) \\
FC $(512)$ & FC (512) & FC (512) \\
ReLU & ReLU & LeakyReLU (0.2) \\
FC $(512)$ & FC $(512)$ & FC (512) \\
ReLU & ReLU & LeakyReLU (0.2) \\
FC $(512)$ & FC $(512)$ & FC (512) \\
ReLU & ReLU & LeakyReLU (0.2) \\
FC $(\operatorname{dim}(x))$ & FC $(\operatorname{dim}(x))$ & FC (1) \\
Sigmoid & Identity & Identity \\
\hline
\end{tabular}

Table 4: Network architecutre of WGANGP on MIMIC-III and Instagram.

\section{B.3 Implementation of Baseline Attacks}

We provide more details of implementing baseline attacks that are discussed in Section 6.7.

B.3.1 LOGAN. For CelebA, we employ DCGAN as the attack model, which is the same as in the original paper [25]. For MIMIC-III and Instagram, we use WGANGP as the attack model.

B.3.2 MC. For implementing MC in the full black-box setting on CelebA, we apply the same process of their best attack on the RGB image dataset: First, we employ principal component analysis (PCA) on a data subset disjoint from the query data. Then, we keep the first 120 PCA components as suggested in the original paper [29] and apply dimensionality reduction on the generated and query data. Finally, we calculate the Euclidean distance of the projected data and use the median heuristic to choose the threshold for MC attack.

\section{ADDITIONAL RESULTS}

\section{C.1 Sanity-check in the White-box Setting}

C.1.1 Analysis on optimization initialization. Due to the non-convexity of our optimization problem, the choice of initialization is of great importance. We explore three different initialization heuristics in

https://github.com/tkarras/progressive_growing_of_gans, https://github.com/igul222/improved_wgan_training, https://github.com/carpedm20/DCGAN-tensorflow, https://github.com/mp2893/medgan,

https://drive.google.com/drive/folders/10RCFaA8kOgkRHXIJpXIWACuUyLiEhlY our experiments, including mean $\left(z_{0}=\mu\right)$, random $\left(z_{0} \sim \mathcal{N}(\mu, \Sigma)\right)$, and nearest neighbour $\left(z_{0}=\operatorname{argmin}_{z \in\left\{z_{i}\right\}_{i=1}^{k}}\left\|\mathcal{G}_{v}(z)-x\right\|_{2}^{2}\right)$. We find that the mean and nearest neighbor initializations perform well in practice, and are in general better than random initialization in terms of the successful reconstruction rate (reconstruction error smaller than 0.01). Therefore, we apply the mean and nearest neighbor initialization in parallel, and choose the one with smaller reconstruction error for the attack.

C.1.2 Analysis on Optimization Method. We explore three optimizers with a range of hyper-parameter search: Adam [39], RMSProp [63], and L-BFGS [46] for reconstructing generated samples of PGGAN on CelebA. Figure 13 shows that L-BFGS achieves superior convergence rate with no additional hyper-parameter. Therefore, we select L-BFGS as our default optimizer in the white-box setting.

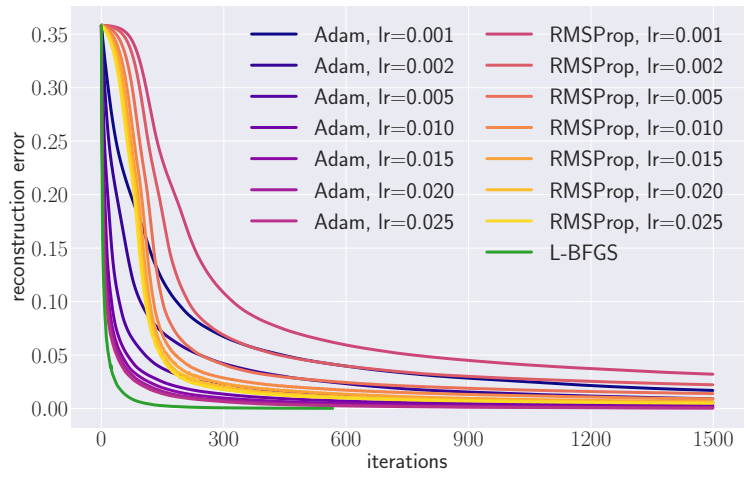

Figure 13: Convergence rate of various optimizers (Adam, RMSProp, L-BFGS) with different learning rates. Mean initialization $\left(z_{0}=\mu\right)$ is applied in this analysis study.

C.1.3 Analysis on Distance Metric Design for Optimization. We show the effectiveness of our objective design (Equation 8). Although optimizing only for element-wise difference term $L_{2}$ yields reasonably good reconstruction in most cases, we observe undesired blur in reconstruction for CelebA images. Incorporating deep image feature term $L_{\text {lpips }}$ and regularization term $L_{\text {reg }}$ benefits the successful reconstruction rate. See Figure 14 for a demonstration.

Table 5: Successful reconstruction rate for generated samples from different GANs.

\begin{tabular}{lcccc}
\hline & DCGAN & PGGAN & WGANGP & VAEGAN \\
\hline Success rate $(\%)$ & 99.89 & 99.83 & 99.55 & 99.25 \\
\hline
\end{tabular}

C.1.4 Sanity Check on Distance Metric Design for Optimization. In addition, we check if the non-convexity of our objective function affects the feasibility of attack against different victim GANs. We apply optimization to reconstruct generated samples. Ideally, the reconstruction should have no error because the query samples are directly generated by the model, i.e., their preimages exist. We set a 


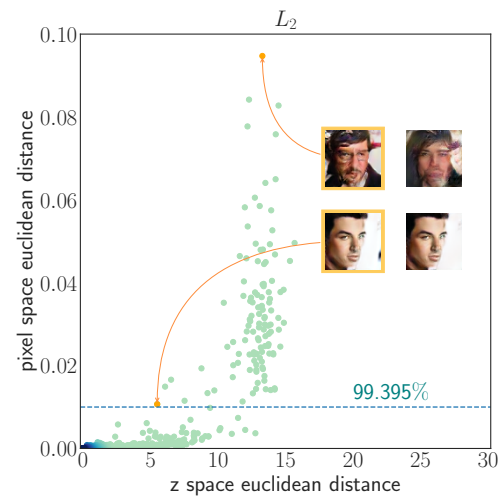

(a)

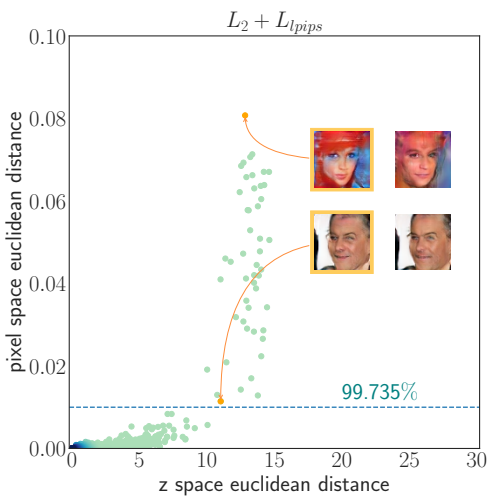

(b)

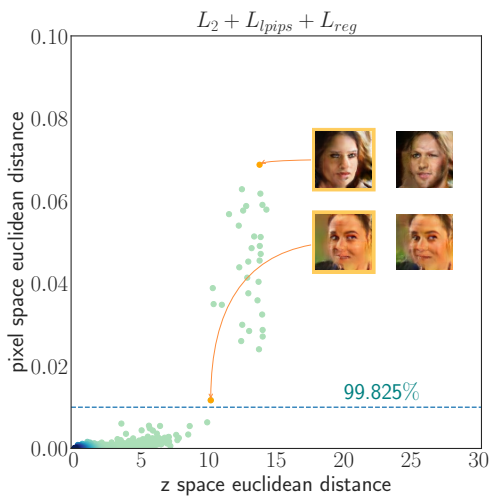

(c)

Figure 14: Reconstruction error plots of PGGAN-generated samples on CelebA. The $x$-axis represents the Euclidean distance between a reconstructed latent code to its ground truth value. The y-axis represents the $L_{2}$ residual in the image domain. The images in orange frame are generated samples. Their reconstructed copies are shown on their right. Samples below the dashed line have reconstruction residuals smaller than 0.01 , where no visual difference can be observed. Therefore, the reconstruction is in general better if there is a higher portion of sample points below the dashed line (a higher successful reconstruction rate). (a) Reconstruction results when disabling $L_{\text {lpips }}$ and $L_{\text {reg }}\left(\lambda_{1}=1.0, \lambda_{2}=0, \lambda_{3}=0\right)$. (b) Reconstruction results when disabling $L_{\text {reg }}$ $\left(\lambda_{1}=1.0, \lambda_{2}=0.2, \lambda_{2}=0\right)$. (c) Reconstruction results when enabling all the $L_{2}, L_{\text {lpips }}$ and $L_{\text {reg }}$ terms $\left(\lambda_{1}=1.0, \lambda_{1}=0.2, \lambda_{2}=0.001\right)$. We find that using all the terms most benefits the reconstruction.

threshold of 0.01 to the reconstruction error for counting successful reconstruction rate, and evaluate the success rate for four GAN models trained on CelebA. Table 5 shows that we obtained more than $99 \%$ success rate for all the GANs, which verifies the feasibility of our optimization-based attack.

C.1.5 Analysis on Distance Metric Design for Classification. We propose to enable/disable $\lambda_{1}, \lambda_{2}$, or $\lambda_{3}$ in Equation 8 to investigate the contribution of each term towards classification thresholding (membership inference) on CelebA. In detail, we consider using (1) the element-wise difference term $L_{2}$ only, (2) the deep image feature term $L_{\text {lpips }}$ only, and (3) all the three terms together to evaluate attack performance. Figure 15 shows the AUCROC of attack against each various GANs. We find that our complete distance metric design achieves general superiority to single terms. Therefore, we use the complete distance metric for classification thresholding.

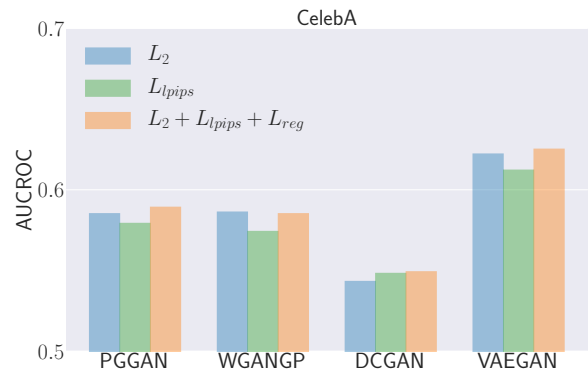

Figure 15: White-box attack performance against GANs on CelebA, w.r.t. distance metric design for classification.

\section{C.2 Additional Quantitative Results}

C.2.1 Evaluation on Full Black-box Attack. Attack Performance w.r.t. Training Set Size: Table 6 corresponds to Figure 5(a), Figure 5(b), and Figure 5(c) in the main paper.

(a) CelebA

\begin{tabular}{lcccccccc}
\hline & 64 & 128 & 256 & 512 & 1024 & 2048 & 4096 & $20 \mathrm{k}$ \\
\hline PGGAN & 1.00 & 1.00 & 1.00 & 0.99 & 0.95 & 0.79 & 0.58 & 0.51 \\
WGANGP & 1.00 & 1.00 & 1.00 & 0.97 & 0.89 & 0.72 & 0.62 & 0.51 \\
\hline
\end{tabular}

(b) MIMIC-III

\begin{tabular}{ccccccccc}
\hline & 64 & 128 & 256 & 512 & 1024 & 2048 & 4096 & 8192 \\
\hline WGANGP & 0.98 & 0.97 & 0.93 & 0.87 & 0.81 & 0.68 & 0.54 & 0.52 \\
MEDGAN & 0.78 & 0.65 & 0.57 & 0.54 & 0.52 & 0.52 & 0.51 & 0.51 \\
\hline
\end{tabular}

(c) Instagram

\begin{tabular}{cccccccccc}
\hline & 64 & 128 & 256 & 512 & 1024 & 2048 & 4096 & 8192 & $10 \mathrm{k}$ \\
\hline WGANGP & 1.00 & 1.00 & 0.97 & 0.90 & 0.72 & 0.54 & 0.50 & 0.50 & 0.50 \\
\hline
\end{tabular}

Table 6: Full black-box attack performance w.r.t. training set size.

Attack Performance w.r.t. Training Set Selection: Table 7 corresponds to Figure 6 in the main paper.

C.2.2 Evaluation on Partial Black-box Attack. Attack Performance w.r.t. Training Set Selection: Table 7 corresponds to Figure 6 in the main paper. 
(a) Full black-box

\begin{tabular}{lcccc}
\hline & PGGAN & WGANGP & DCGAN & VAEGAN \\
\hline random & 0.51 & 0.51 & 0.51 & 0.50 \\
identity & 0.53 & 0.53 & 0.51 & 0.51 \\
\hline
\end{tabular}

(b) Partial black-box

\begin{tabular}{ccccc}
\hline & PGGAN & WGANGP & DCGAN & VAEGAN \\
\hline random & 0.55 & 0.53 & 0.51 & 0.55 \\
identity & 0.57 & 0.60 & 0.55 & 0.58 \\
\hline
\end{tabular}

(c) White-box

\begin{tabular}{ccccc}
\hline & PGGAN & WGANGP & DCGAN & VAEGAN \\
\hline random & 0.54 & 0.53 & 0.52 & 0.61 \\
identity & 0.59 & 0.59 & 0.55 & 0.63 \\
\hline
\end{tabular}

Table 7: Attack performance on the random v.s. identitybased GAN training set selection. We only focus on CelebA across attack settings.

C.2.3 Evaluation on White-box Attack. Ablation on Distance Metric Design for Classification: Table 8 corresponds to Figure 15.

\begin{tabular}{lcccc}
\hline & DCGAN & PGGAN & WGANGP & VAEGAN \\
\hline$L_{2}$ & 0.54 & 0.59 & 0.59 & 0.62 \\
$L_{\text {lpips }}$ & 0.55 & 0.58 & 0.57 & 0.61 \\
$L_{2}+L_{\text {lpips }}+L_{\text {reg }}$ & 0.55 & 0.59 & 0.59 & 0.63 \\
\hline
\end{tabular}

Table 8: White-box attack performance against various GANs on CelebA, w.r.t. distance metric design for classification.

Attack Performance w.r.t. Training Set Size: Table 9 corresponds to Figure 5(d), Figure 5(e), and Figure 5(f).

Attack Performance w.r.t. Training Set Selection: Table 7 corresponds to Figure 6 in the main paper.

C.2.4 Attack Calibration. Table 10 corresponds to Figure 7 in the main paper. Table 11 corresponds to Figure 8 in the main paper.

C.2.5 Comparison to Baseline Attacks. Table 12 corresponds to Figure 9 in the main paper. Table 13 corresponds to Figure 11 in the main paper. Table 14 corresponds to Figure 10 in the main paper. (a) CelebA

\begin{tabular}{lcccccccc}
\hline & 64 & 128 & 256 & 512 & 1024 & 2048 & 4096 & $20 \mathrm{k}$ \\
\hline PGGAN & 1.00 & 1.00 & 1.00 & 0.99 & 0.95 & 0.83 & 0.62 & 0.55 \\
WGANGP & 1.00 & 1.00 & 0.99 & 0.97 & 0.89 & 0.78 & 0.69 & 0.53 \\
\hline
\end{tabular}

(b) MIMIC-III

\begin{tabular}{ccccccccc}
\hline & 64 & 128 & 256 & 512 & 1024 & 2048 & 4096 & 8192 \\
\hline WGANGP & 0.98 & 0.96 & 0.92 & 0.87 & 0.82 & 0.80 & 0.67 & 0.54 \\
MEDGAN & 0.99 & 0.88 & 0.77 & 0.72 & 0.61 & 0.59 & 0.55 & 0.52 \\
\hline
\end{tabular}

(c) Instagram

\begin{tabular}{cccccccccc}
\hline & 64 & 128 & 256 & 512 & 1024 & 2048 & 4096 & 8192 & $10 \mathrm{k}$ \\
\hline WGANGP & 1.00 & 1.00 & 0.97 & 0.90 & 0.72 & 0.55 & 0.50 & 0.50 & 0.49
\end{tabular}

Table 9: White-box attack performance w.r.t. training set size.

(a) Full black-box

\begin{tabular}{lcccc}
\hline & PGGAN & WGANGP & DCGAN & VAEGAN \\
\hline before calibration & 0.53 & 0.53 & 0.51 & 0.51 \\
after calibration & 0.54 & 0.54 & 0.52 & 0.51 \\
\hline
\end{tabular}

(b) Partial black-box

\begin{tabular}{lcccc}
\hline & PGGAN & WGANGP & DCGAN & VAEGAN \\
\hline before calibration & 0.57 & 0.60 & 0.55 & 0.58 \\
after calibration & 0.58 & 0.63 & 0.56 & 0.59 \\
\hline
\end{tabular}

(c) White-box

\begin{tabular}{lcccc}
\hline & PGGAN & WGANGP & DCGAN & VAEGAN \\
\hline before calibration & 0.59 & 0.59 & 0.55 & 0.63 \\
after calibration & 0.68 & 0.64 & 0.55 & 0.76 \\
\hline
\end{tabular}

Table 10: Attack performance before and after calibration on CelebA.

\begin{tabular}{lccccc}
\hline & PGGAN & WGANGP & DCGAN & VAEGAN & VAE \\
\hline full bb (LOGAN) & 0.56 & 0.57 & 0.52 & 0.50 & 0.52 \\
full bb (MC) & 0.52 & 0.52 & 0.51 & 0.50 & 0.51 \\
full bb (ours calibrated) & 0.54 & 0.54 & 0.52 & 0.51 & 0.54 \\
partial bb (ours calibrated) & 0.58 & 0.63 & 0.56 & 0.59 & 0.73 \\
wb (ours calibrated) & 0.68 & 0.66 & 0.55 & 0.76 & 0.94 \\
full (LOGAN/MC) & 0.91 & 0.83 & 0.83 & 0.61 & 0.90 \\
\hline
\end{tabular}

Table 12: Comparison of different attacks on CelebA. bb: black-box; wb: white-box; full: accessible discriminator (full model). 
(a) MIMIC-III (WGANGP)

\begin{tabular}{lcccccccc}
\hline & 64 & 128 & 256 & 512 & 1024 & 2048 & 4096 & 8192 \\
\hline full bb & 0.98 & 0.97 & 0.93 & 0.87 & 0.81 & 0.68 & 0.54 & 0.52 \\
full bb (calibrated) & 1.00 & 0.99 & 0.97 & 0.94 & 0.89 & 0.84 & 0.67 & 0.56 \\
wb & 0.98 & 0.96 & 0.92 & 0.87 & 0.82 & 0.80 & 0.67 & 0.54 \\
wb (calibrated) & 0.98 & 0.97 & 0.93 & 0.90 & 0.87 & 0.85 & 0.75 & 0.59 \\
\hline
\end{tabular}

(b) MIMIC-III (MEDGAN)

\begin{tabular}{lcccccccc}
\hline & 64 & 128 & 256 & 512 & 1024 & 2048 & 4096 & 8192 \\
\hline full bb & 0.78 & 0.65 & 0.57 & 0.54 & 0.52 & 0.52 & 0.51 & 0.51 \\
full bb (calibrated) & 0.91 & 0.71 & 0.63 & 0.58 & 0.55 & 0.53 & 0.52 & 0.51 \\
wb & 0.99 & 0.88 & 0.77 & 0.72 & 0.61 & 0.59 & 0.55 & 0.52 \\
wb (calibrated) & 0.96 & 0.87 & 0.81 & 0.75 & 0.65 & 0.62 & 0.57 & 0.55 \\
\hline
\end{tabular}

(c) Instagram (WGANGP)

\begin{tabular}{lccccccccc}
\hline & 64 & 128 & 256 & 512 & 1024 & 2048 & 4096 & 8192 & $10 \mathrm{k}$ \\
\hline full bb & 1.00 & 1.00 & 0.97 & 0.90 & 0.72 & 0.54 & 0.50 & 0.50 & 0.49 \\
full bb (calibrated) & 1.00 & 1.00 & 0.98 & 0.91 & 0.80 & 0.72 & 0.65 & 0.57 & 0.56 \\
wb & 1.00 & 1.00 & 0.97 & 0.90 & 0.72 & 0.55 & 0.50 & 0.50 & 0.49 \\
wb (calibrated) & 1.00 & 1.00 & 0.98 & 0.92 & 0.79 & 0.73 & 0.67 & 0.58 & 0.57
\end{tabular}

Table 11: Attack performance before and after calibration for non-image datasets w.r.t. GAN training set sizes. bb: black-box; wb: white-box.

\begin{tabular}{lcccccccccccccc}
\hline$k$ & 64 & 128 & 256 & 512 & 1024 & 2048 & 4096 & 8192 & $15 \mathrm{k}$ & $20 \mathrm{k}$ & $40 \mathrm{k}$ & $60 \mathrm{k}$ & $80 \mathrm{k}$ & $100 \mathrm{k}$ \\
\hline LOGAN & 0.51 & 0.51 & 0.51 & 0.52 & 0.53 & 0.53 & 0.53 & 0.54 & 0.55 & 0.56 & 0.57 & 0.58 & 0.57 & 0.57 \\
MC & 0.50 & 0.50 & 0.51 & 0.51 & 0.51 & 0.51 & 0.52 & 0.52 & 0.52 & 0.52 & 0.52 & 0.53 & 0.53 & 0.53
\end{tabular}
$\begin{array}{lllllllllllllll}\text { MC } & 0.50 & 0.50 & 0.51 & 0.51 & 0.51 & 0.51 & 0.52 & 0.52 & 0.52 & 0.52 & 0.52 & 0.53 & 0.53 & 0.53\end{array}$ \begin{tabular}{ccccccccccccccc} 
ours calibrated & 0.51 & 0.51 & 0.51 & 0.52 & 0.52 & 0.52 & 0.53 & 0.53 & 0.54 & 0.54 & 0.54 & 0.55 & 0.55 & 0.55 \\
\hline Table 14: Full black-box attack performance against PGGAN
\end{tabular} on CelebA w.r.t. $k$ in Equation 5, the number of generated samples.

C.2.6 Defense. Table 15 corresponds to Figure 12(a) in the main paper. Table 16 corresponds to Figure 12(b) in the main paper.

\begin{tabular}{lccc}
\hline & full black-box & partial black-box & white-box \\
\hline w/o DP & 0.54 & 0.58 & 0.68 \\
w/ DP & 0.53 & 0.56 & 0.59
\end{tabular}

Table 15: Attack performance against PGGAN on CelebA with or without DP defense.

\begin{tabular}{lccccccc}
\hline & 64 & 128 & 256 & 512 & 1024 & 2048 & 4096 \\
\hline white-box w/o DP & 1.00 & 1.00 & 1.00 & 0.99 & 0.95 & 0.83 & 0.62 \\
white-box w/ DP & 1.00 & 1.00 & 0.99 & 0.98 & 0.90 & 0.70 & 0.56 \\
full black-box w/o DP & 1.00 & 1.00 & 1.00 & 0.99 & 0.95 & 0.79 & 0.57 \\
full black-box w/ DP & 1.00 & 1.00 & 0.99 & 0.98 & 0.89 & 0.68 & 0.53
\end{tabular}

Table 16: Attack performance against PGGAN on CelebA with or without DP defense, w.r.t. GAN training set size.

\section{C.3 Additional Qualitative Results}

Given query samples $x$, we show their reconstruction copies $R\left(x \mid \mathcal{G}_{v}\right)$ and $R\left(x \mid \mathcal{G}_{r}\right)$ obtained in our white-box attack.

\begin{tabular}{lcccccccc}
\hline & 64 & 128 & 256 & 512 & 1024 & 2048 & 4096 & 8192 \\
\hline full bb (LOGAN) & 0.98 & 0.97 & 0.96 & 0.94 & 0.92 & 0.83 & 0.65 & 0.54 \\
full bb (ours calibrated) & 1.00 & 0.99 & 0.97 & 0.94 & 0.89 & 0.84 & 0.67 & 0.56 \\
wb (ours calibrated) & 0.98 & 0.97 & 0.93 & 0.90 & 0.87 & 0.85 & 0.75 & 0.59 \\
dis (LOGAN) & 1.00 & 1.00 & 1.00 & 1.00 & 1.00 & 1.00 & 0.99 & 0.98 \\
\hline
\end{tabular}

(b) MIMIC-III (MEDGAN)

\begin{tabular}{lcccccccc}
\hline & 64 & 128 & 256 & 512 & 1024 & 2048 & 4096 & 8192 \\
\hline full bb (LOGAN) & 0.45 & 0.57 & 0.53 & 0.52 & 0.51 & 0.52 & 0.50 & 0.51 \\
full bb (ours calibrated) & 0.91 & 0.71 & 0.63 & 0.58 & 0.55 & 0.53 & 0.52 & 0.51 \\
wb (calibrated) & 0.96 & 0.87 & 0.81 & 0.75 & 0.65 & 0.62 & 0.57 & 0.55 \\
dis (LOGAN) & 1.00 & 0.92 & 0.96 & 0.90 & 0.85 & 0.90 & 0.80 & 0.73 \\
\hline
\end{tabular}

(c) Instagram (WGANGP)

\begin{tabular}{lccccccccc}
\hline & 64 & 128 & 256 & 512 & 1024 & 2048 & 4096 & 8192 & $10 \mathrm{k}$ \\
\hline full bb (LOGAN) & 1.00 & 0.99 & 0.96 & 0.91 & 0.68 & 0.55 & 0.58 & 0.55 & 0.55 \\
full bb (calibrated) & 1.00 & 1.00 & 0.98 & 0.91 & 0.80 & 0.72 & 0.65 & 0.57 & 0.56 \\
wb (calibrated) & 1.00 & 1.00 & 0.98 & 0.92 & 0.79 & 0.73 & 0.67 & 0.58 & 0.57 \\
dis (LOGAN) & 1.00 & 1.00 & 1.00 & 1.00 & 1.00 & 1.00 & 0.98 & 0.96 & 0.93 \\
\hline
\end{tabular}

Table 13: Comparison of different attacks on the other two non-image datasets w.r.t. GAN training set size. bb: blackbox; wb: white-box; dis: accessible discriminator. 


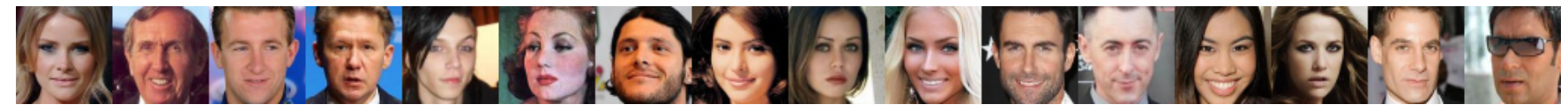

(a) Query (real) images

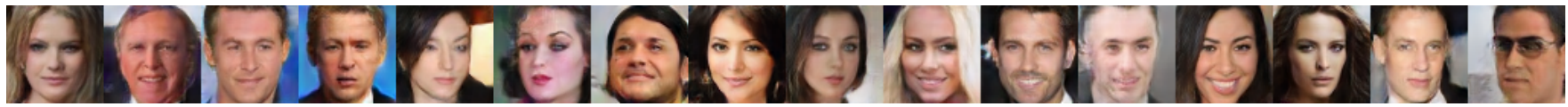

(b) PGGAN victim model reconstruction

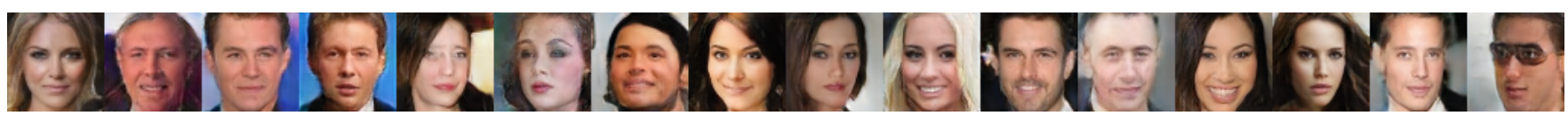

(c) PGGAN (w/ DP) victim model reconstruction

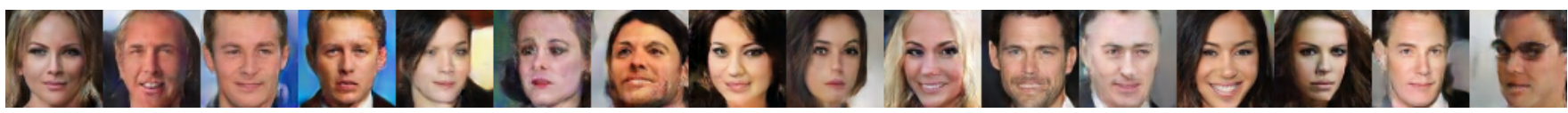

(d) PGGAN reference model reconstruction

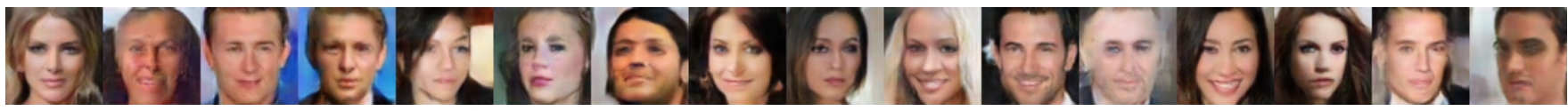

(e) WGANGP victim model reconstruction

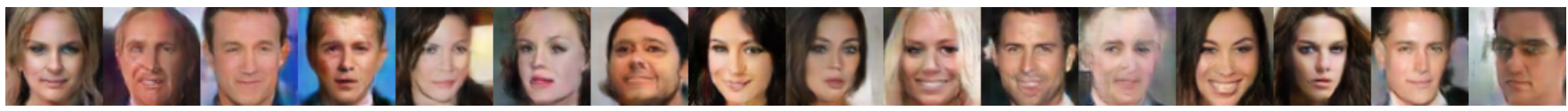

(f) WGANGP reference model reconstruction

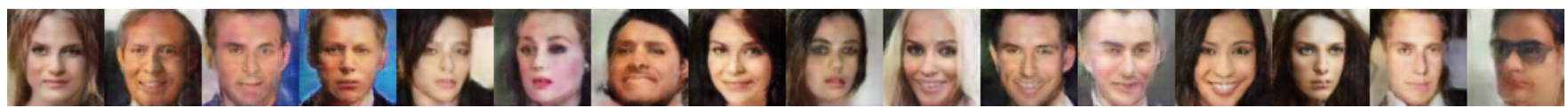

(g) DCGAN victim model reconstruction

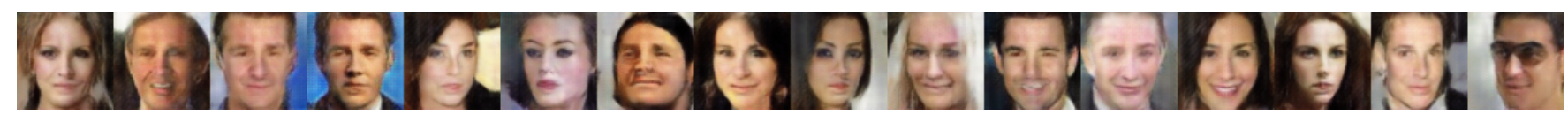

(h) DCGAN reference model reconstruction

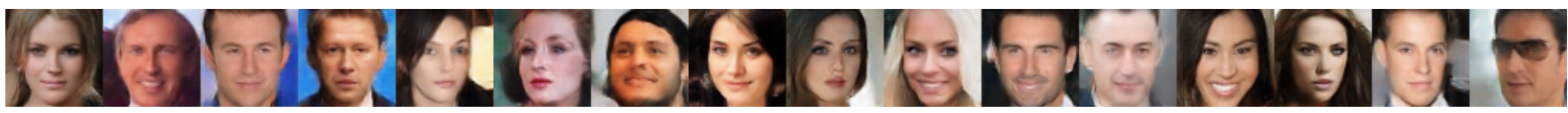

(i) VAEGAN victim model reconstruction

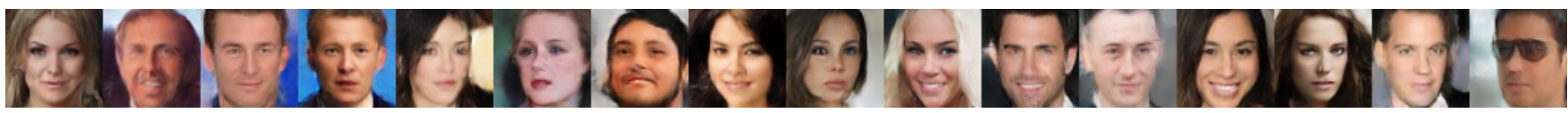

(j) VAEGAN reference model reconstruction

Figure 16: Reconstruction of query samples $x$ that are in the training set, i.e., $x \in D_{\text {train }}$. 


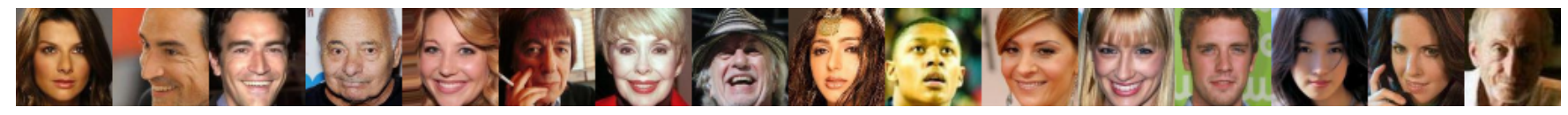

(a) Query (real) images

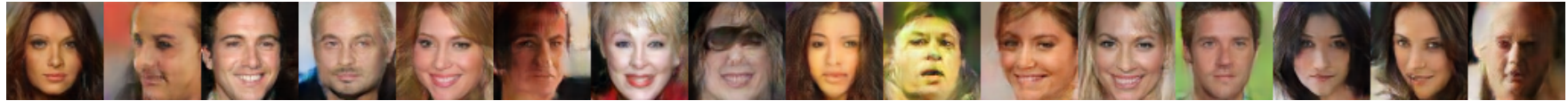

(b) PGGAN victim model reconstruction

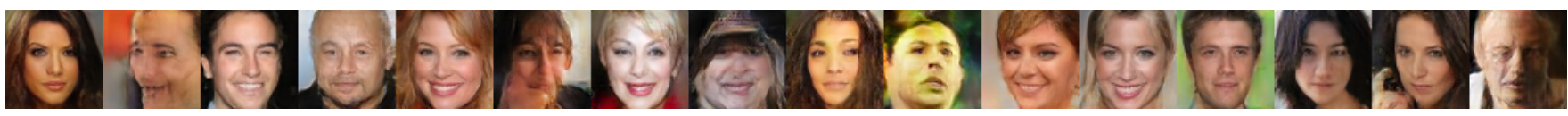

(c) PGGAN (w/DP) victim model reconstruction

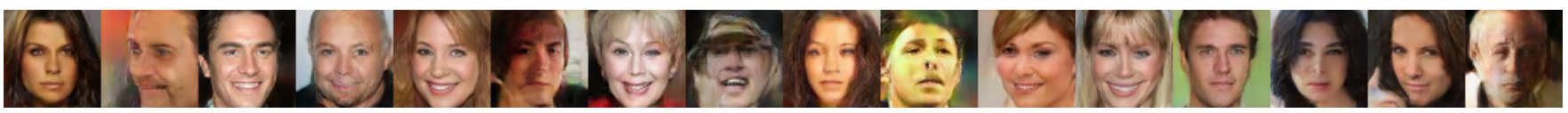

(d) PGGAN reference model reconstruction

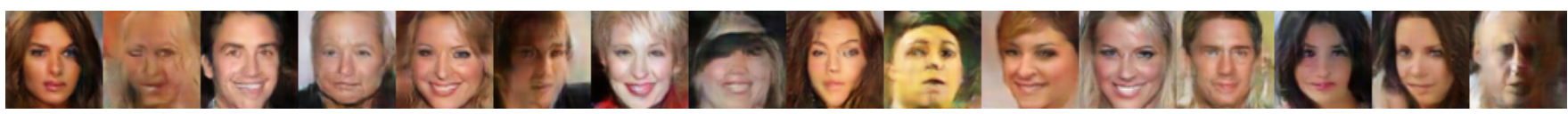

(e) WGANGP victim model reconstruction

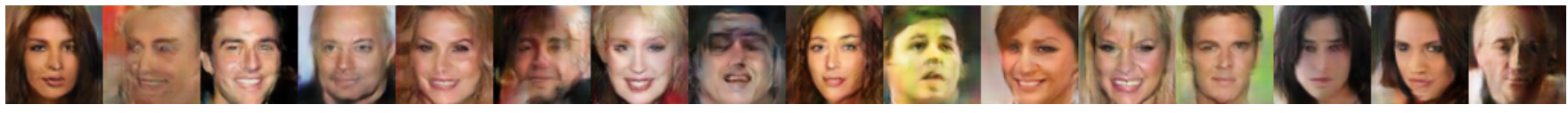

(f) WGANGP reference model reconstruction

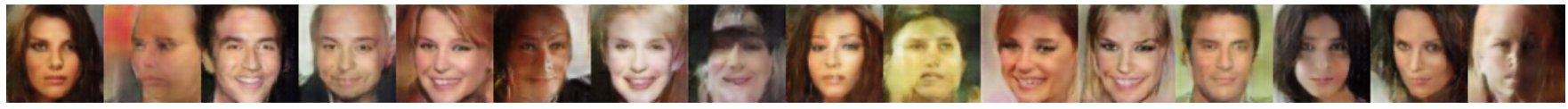

(g) DCGAN victim model reconstruction

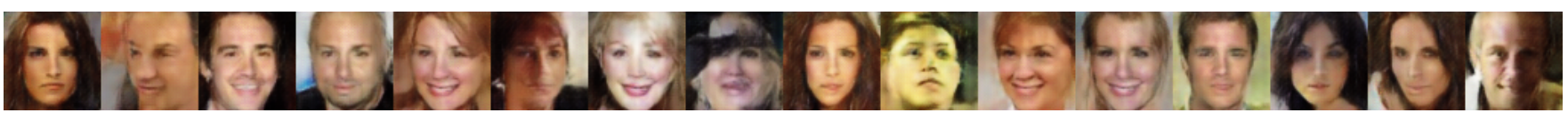

(h) DCGAN reference model reconstruction

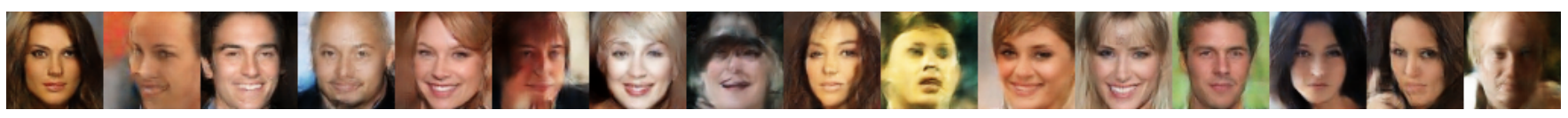

(i) VAEGAN victim model reconstruction

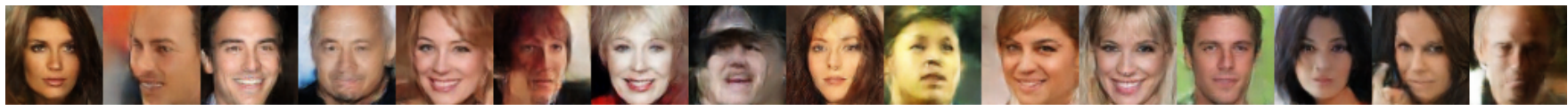

(j) VAEGAN reference model reconstruction

Figure 17: Reconstruction of query samples $x$ that are not in the training set, i.e., $x \notin D_{\text {train }}$. 